\title{
Sampling-Based Event-Triggered Control for Neutral-Type Complex-Valued Neural Networks with Partly Unknown Markov Jump and Time-Varying Delay
}

\author{
Zhen Wang $\mathbb{D}^{D}$, Lianglin Xiong $\mathbb{D}$, Haiyang Zhang $\mathbb{D}^{\text {, }}$, and Yingying Liu \\ School of Mathematics and Computer Science, Yunnan Minzu University, Kunming 650500, China \\ Correspondence should be addressed to Lianglin Xiong; lianglin_5318@126.com
}

Received 6 January 2021; Accepted 10 May 2021; Published 24 May 2021

Academic Editor: Rui Wang

Copyright (c) 2021 Zhen Wang et al. This is an open access article distributed under the Creative Commons Attribution License, which permits unrestricted use, distribution, and reproduction in any medium, provided the original work is properly cited.

\begin{abstract}
This work is devoted to studying the stochastic stabilization of a class of neutral-type complex-valued neural networks (CVNNs) with partly unknown Markov jump. Firstly, in order to reduce the conservation of our stability conditions, two integral inequalities are generalized to the complex-valued domain. Secondly, a state-feedback controller is designed to investigate the stability of the neutral-type CVNNs with $H \infty$ performance, making the stability problem a further extension, and then, the stabilization of the CVNNs with $H \infty$ performance is investigated through a sampling-based event-triggered (SBET) control for the first time that the transmission event is not triggered except when it violates the event-triggered condition. Finally, two examples are given to illustrate the validity and correctness of our obtained theorems.
\end{abstract}

\section{Introduction}

In terms of the wide application in electromagnetic processing, light wave and sound wave, the neural networks have attracted much attention in recent decades [1-3]. In the meantime, complex-valued signals occur inevitably in practice, and more and more scholars began to make investigations on CVNNs [4-12]. There are two methods usually used in the study of CVNNs: one is to divide the neural networks into the real part and imaginary part, then, the original CVNNs will be changed into real-valued neural networks [8-10]. The other method is when the activation function in CVNNs cannot be separated, the stability condition of the system will be sure by the complex-valued LKFs under the condition that the activation function satisfies the complex-valued Lipschitz continuity $[11,12]$, and it would increase the difficulty of the analysis. Considering the network works which are transformed in the first method are real-valued systems, it is easier to understand. Unfortunately, the dimensions of the obtained real-valued neural networks are twice the dimension of the original CVNNs. Also, the partial derivatives of the real and imaginary parts of state variables of the activation function are required not only to exist but also to be continuous and bounded, resulting in problems in our analysis and the application of the obtained conclusions would be limited. According to the state of the art, the LKFs method is often used to deal with neural networks problems because of its simplicity and effectiveness [10, 13-15], so it is necessary to construct LKFs with conjugate transpose of state vector to study the CVNNs by the method which do not separate the original system.

Due to the complexity of the reality, certain systems are sometimes difficult to apply to the reality, making the research of uncertain systems more valuable and practical [16]. $H \infty$ control provides a good method of dealing with unstructured uncertain systems, and research related has grown over the past two decades. Besides, it was increasingly used to analyse problems in the field of robotics, aerospace, and power systems $[13,16-20]$. Reference [16] was concerned with the stabilization problem for uncertain T-S fuzzy systems with time-varying delays via a robust $H \infty$ state-feedback controller. And more general LKFs method with relaxed conditions was constructed through an improved time-delay interval segmentation method. The 
state-feedback $H \infty$ control problem of time-delay systems is studied in [13], and the reciprocal convex inequality was used to obtain stability conditions of the system. However, the research we mentioned is all committed to the real systems; there is little related research on the $H \infty$ performance of CVNNs, and it is one of the main tasks in this paper. Furthermore, many scholars have specialized in the dynamic system with Markov jumping on account of the universal of Markov phenomenon, and abundant achievements have been made [14, 21-27]. In the applications of practical engineering, the analysis and control synthesis of Markov jumping systems are troubled by many dicey factors, such as the partial unknown transmission probability and the uncertainty of transmission rates. Some preliminary results have been obtained in the study of Markov jumping systems with partially unknown transmission probability $[21,23,24,27]$. To our knowledge, less effort has been made on CVNNs with Markov phenomenon. To sum up, it is necessary to analyze the stability and $H \infty$ performance of Markov jumping CVNNs in the case of partially unknown transmission probability.

In the past few years, the sampled-data control and event-triggered control as a discrete control have attracted much attention from scholars $[15,28-40]$. The so-called event-triggered control refers to a control of the tasks; whether to be executed is determined by the given eventtriggered conditions in advance rather than according to the time. The control tasks would execute $d$ immediately when the event-triggered mechanism is broken out. Comparing to the controller with time mechanism [32], events-triggered control can save the computing resources, battery energy, and communication resources obviously. Note that the event-triggered was continuous in the inchoate phase and that special hardware is needed to monitor the status continuously. Yue dong et al. proposed the (SBET) control, which is a discrete one [34]. And the monitor only needs to observe the state of the system at discrete instants with a
SBET scheme that can effectively reduce the number of control tasks and save the communication resources significantly. In literature [37], the global asymptotic stability of the CVNNs under the framework of event-triggered control was studied by dividing the system into real and imaginary parts. There is an output-feedback $H \infty$ control under the event-triggered framework with nonuniform sampling used to explain the stability of networked control systems by Peng and Zheng [33]. Unfortunately, there is almost no research on CVNNs with a SBET control. So, how to stabilize the CVNNs by designing a sampling-based event-triggered controller is of our interest.

To date, this paper focuses on the stabilization of CVNNs with partly unknown Markov jump and time-varying delay. Firstly, a $H \infty$ state-feedback control is proposed to explain the stability of neutral-type CVNNs; to our knowledge, there is little research about the stabilization of neural networks in the complex field. Secondly, the stabilization of Markov jumping neutral-type CVNNs with $H \infty$ performance is considered under the framework of a SBET controller. And it is the first time to study CVNNs with a sampling-based event-triggered mechanism while avoid splitting the system into two parts, which reduces the computational complexity greatly.

Notations: throughout this work, $\mathscr{C}$ denotes the complex field and $\mathscr{C}^{n}$ is the $n$ dimensional complex space. $\mathscr{A}>0$ (or $\mathscr{A}<0$ ) means that $\mathscr{A}$ is a positive Hermitian matrix (or negative Hermitian matrix), $\operatorname{sym}\{\mathscr{A}\}=\mathscr{A}^{H}+\mathscr{A}$, where $\mathscr{A}^{H}$ and $\mathscr{A}^{T}$, respectively, mean the conjugate transpose matrix and transpose matrix of $\mathscr{A}, *$ in a matrix denotes the selfconjugate part of the Hermitian matrix.

\section{Problem Description and Preliminaries}

2.1. Problem Description. Consider a class of neutral-type CVNNs with partly unknown Markov jump as follows:

$$
\left\{\begin{array}{l}
\dot{\mathcal{\vartheta}}(t)-\mathscr{B}_{1}\left(r_{t}\right) \dot{\mathfrak{\vartheta}}(t-\sigma(t))=-\mathscr{A}_{1}\left(r_{t}\right) \mathcal{\vartheta}(t)+\mathscr{C}_{1}\left(r_{t}\right) \varphi(\mathcal{\vartheta}(t))+\mathscr{D}_{1}\left(r_{t}\right) \varphi(\vartheta(t-\sigma(t)))+\mathscr{E}_{1}\left(r_{t}\right) \omega(t)+\mathscr{E}_{2}\left(r_{t}\right) u(t) \\
v(t)=-\mathscr{A}_{2}\left(r_{t}\right) \mathcal{\vartheta}(t)+\mathscr{C}_{2}\left(r_{t}\right) \varphi(\vartheta(t))+\mathscr{D}_{2}\left(r_{t}\right) \varphi(\vartheta(t-\sigma(t)))+\mathscr{H}_{1}\left(r_{t}\right) \omega(t), \\
\mathfrak{\vartheta}\left(t_{0}+\theta\right)=\psi(\theta), \quad \forall \theta \in[-\sigma, 0],
\end{array}\right.
$$

where $\vartheta(t) \in \mathscr{C}^{n}$ is the state vector, which is described as $\left(\vartheta_{1}(t), \vartheta_{2}(t), \ldots, \vartheta_{j}(t)\right)$ and $\vartheta_{j}(t)$ expresses the state of the jth neuron. $\omega(t) \in \mathscr{C}^{n}$ is the external disturbance, $u(t) \in \mathscr{C}^{n}$ is a control input, $v(t) \in \mathscr{C}^{m}$ is the control output, $0 \leq \sigma(t) \leq \sigma, \dot{\sigma}(t) \leq \sigma_{d}$ is the time-varying delay, and $\varphi(\theta) \in \mathscr{C}^{n}$ is the initial value condition. $\varphi(\vartheta(t))=\operatorname{col}\left\{\varphi_{1}(\vartheta(t)), \varphi_{2}(\mathcal{Y}(t)), \ldots, \varphi_{n}(\vartheta(t))\right\} \in \mathscr{C}^{n}, \quad\left\{r_{t}\right\}$ denotes a Markov process with on the probability space $t \geq 0$, which is right-continuous, and it takes values in set $\wp=$ $\{1,2, \ldots, n\}$ with the transition rate matrix $\Pi=\left(\pi_{p q}\right), p, q \in \wp$ given by

$$
\operatorname{Pr}\left\{r_{t+\Delta}=q \mid r_{t}=p\right\}= \begin{cases}\pi_{p q} \Delta+o(\Delta), & q \neq p, \\ 1+\pi_{p q} \Delta+o(\Delta), & q=p .\end{cases}
$$

However, not all the information of our transition rates can be obtained; then, the transition rate matrix $\Pi$ with $\mathbb{N}$ modes can be expressed as

$$
\Pi=\left(\begin{array}{ccccc}
\pi_{11} & ? & \pi_{13} & \cdots & ? \\
? & ? & ? & \cdots & \pi_{2 n} \\
\vdots & \vdots & \vdots & \ddots & \vdots \\
? & \pi_{n 2} & \pi_{n 3} & \cdots & \pi_{n n}
\end{array}\right),
$$


where? expresses the transition rates which are unknown and, for $\forall p \in \wp$, the set $\mathscr{P}^{p}$ is expressed as $\mathscr{P}^{p}=\mathscr{P}_{k}^{p} \bigcup \mathscr{P}^{p}{ }^{p}$ with

$$
\begin{aligned}
\aleph_{k}^{p} \triangleq\left\{q: \pi_{p q} \text { is known for } q \in \wp\right\}, \\
\aleph_{u k}^{p} \triangleq\left\{q: \pi_{p q} \text { is uknown for } q \in \wp\right\} .
\end{aligned}
$$

With $r_{t}=p$ and the controllers being considered as $u(t)=\mathscr{K}_{p} \vartheta(t)$, then system (1) would be reexpressed as

$$
\left\{\begin{array}{l}
\dot{\vartheta}(t)=\left(-\mathscr{A}_{1 p}+\mathscr{E}_{2 p} \mathscr{K}_{p}\right) \vartheta(t)+\mathscr{B}_{1 p} \dot{\vartheta}(t-\sigma(t))+\mathscr{C}_{1 p} \varphi(\vartheta(t))+\mathscr{D}_{1 p} \varphi(\vartheta(t-\sigma(t)))+\mathscr{E}_{1 p} \omega(t) \\
v(t)=-\mathscr{A}_{2 p} \vartheta(t)+\mathscr{C}_{2 p} \varphi(\vartheta(t))+\mathscr{D}_{2 p} \varphi(\vartheta(t-\sigma(t)))+\mathscr{H}_{1 p} \omega(t) .
\end{array}\right.
$$

2.2. The SBET Scheme. To economize resource, we study the stabilization of the CVNNs (1) under the SBET control in this section. Assuming that the sampling device performs periodically sampling on the system state, $F_{1}=\{0, h, 2 h, \ldots, k h, \ldots\}$ is the sampling sequence with $k \in Z^{+}$, and $h$ is the constant sampling period, set $F_{2}=\left\{0, \iota_{1} h, \iota_{2} h, \ldots, \iota_{k} h, \ldots\right\}, \iota_{k} \in Z^{+}$, describe the eventtriggered sequence, it is clear that $\mathrm{F}_{2} \subseteq \mathrm{F}_{1}$, and the samplingbased event-triggered scheme is given as

$$
\iota_{k+1} h=\iota_{k} h+\inf \left\{l h \mid e^{H}\left(\iota_{k} h+l h\right) \mathscr{G} e\left(\iota_{k} h+l h\right) \geq \omega \vartheta^{H}\left(\iota_{k} h\right) \mathscr{G} \vartheta\left(\iota_{k} h\right)\right\}=\left(\iota_{k}+l_{m}\right) h,
$$

where $l_{m}=(1 / h) \inf \left\{l h \mid e^{H}\left(\iota_{k} h+l h\right) \mathscr{G} e\left(\iota_{k} h+l h\right) \geq \omega \vartheta^{H}\left(\iota_{k}\right.\right.$ h) $\left.\mathscr{G} \vartheta\left(\iota_{k} h\right)\right\}$ and $\mathscr{G}$ is a positive Hermitian matrix, $e\left(l_{k}\right.$ $h+l h)=\vartheta\left(l_{k} h+l h\right)-\vartheta\left(l_{k} h\right)$, with $l \in Z^{+}, 0 \leq \emptyset<1$. As a matter of fact, the network-induced time delay is inevitable when signals are transmitted between the event generator and the actuator at time $t$, which can be expressed as $\varsigma\left(l_{k} h\right)$, and $\varsigma\left(l_{k} h\right) \in[0, \varsigma]$. In $\left[t_{k}, t_{k+1}\right)$, the control signal is hold by a zero-order-hold (ZOH) function. For $j=0,1, \ldots, l_{m-1}$, set $\mathscr{T}_{k}=\left[\left(\iota_{k}+j\right) h+\varsigma\left(\left(\iota_{k}+j\right) h\right)\right.$, $\left.\left(\iota_{k}+j+1\right) h+\varsigma\left(\left(\iota_{k}+j+1\right) h\right)\right]$, and define $\quad \eta(t)=t$ $-\left(\iota_{k}+j\right) h-\varsigma\left(\left(\iota_{k}+j\right) h\right), \quad \eta^{*}(t)=\eta(t)+\varsigma\left(\left(\iota_{k}+j\right) h\right), \quad 0<\eta$ $(t) \leq \eta^{*}(t) \leq \eta=h+\varsigma$, with $t_{k}=\iota_{k} h+\varsigma\left(\iota_{k} h\right)$, and the SBET controllers are represented as

$$
u(t)=\widetilde{\mathscr{K}}_{p} \vartheta\left(\iota_{k} h\right)=\widetilde{\mathscr{K}}_{p} \vartheta\left(t-\eta^{*}(t)\right)-\widetilde{\mathscr{K}}_{p} e\left(t-\eta^{*}(t)\right),
$$

with $t \in \mathscr{T}_{k}$; system (1) would be transformed to

$$
\left\{\begin{array}{l}
\dot{\vartheta}(t)=-\mathscr{A}_{1 p} \vartheta(t)+\mathscr{B}_{1 p} \dot{\vartheta}(t-\sigma(t))+\mathscr{C}_{1 p} \varphi(\vartheta(t))+\mathscr{D}_{1 p} \varphi(\vartheta(t-\sigma(t)))+\mathscr{E}_{1 p} \omega(t)+\mathscr{E}_{2 p} \tilde{\mathscr{K}}_{p} \vartheta\left(t-\eta^{*}(t)\right)-\mathscr{E}_{2 p} \tilde{\mathscr{K}}_{p} e(t-\eta *(t)) \\
v(t)=-\mathscr{A}_{2 p} \vartheta(t)+\mathscr{C}_{2 p} \varphi(\vartheta(t))+\mathscr{D}_{2 p} \varphi(\vartheta(t-\sigma(t)))+\mathscr{H}_{1 p} \omega(t) .
\end{array}\right.
$$

Remark 1 . The parameter $₫$ can make a significant difference in whether the data would be released or not. As $₫$ gets smaller, conditions (6) are more likely to be violated.

Case 1: if $₫=0$, the SBET scheme would be degenerated into

$$
\iota_{k+1} h=\iota_{k} h+\inf \left\{l h \mid e^{H}\left(\iota_{k}+l h\right) \mathscr{G} e\left(\iota_{k}+l h\right) \geq 0\right\},
$$

that all the sampled data would be transmitted, and the SBET scheme (6) reduces to a periodic time-triggered one.

Case 2: if the right-hand side of the inequality is a constant $b>0$, the SBET scheme would be changed into

$$
\iota_{k+1} h=\iota_{k} h+\inf \left\{l h \mid e^{H}\left(\iota_{k}+l h\right) \mathscr{G} e\left(\iota_{k}+l h\right) \geq b\right\}
$$

and then, the SBET scheme (6) is converted from a relative threshold event-trigger condition to a fixed threshold eventtrigger condition. However, an event-triggered control with the condition in case 2 may cause the system to be unstable and fail to achieve the control intention.

The following assumptions are put forward to draw the main results.

Assumption 1. Each activation function $\varphi_{p}(\cdot)$ in (5) satisfies the following condition:

$$
\left|\varphi_{p}\left(\vartheta_{1}\right)-\varphi_{p}\left(\vartheta_{2}\right)\right| \leq l_{p}\left|\vartheta_{1}-\vartheta_{2}\right|
$$

where $\vartheta_{1} \neq \vartheta_{2} \quad$ and $L=\operatorname{diag}\left\{l_{1}, l_{2}, \ldots, l_{n}\right\} \quad$ with $l_{i}>0, i=1,2, \ldots, n$. 
Definition 1 (see [28]). System (5) is said to be stochastically stable if the following inequality holds:

$$
\mathbb{E}\left\{\int_{0}^{\infty}\|\vartheta(s)\|^{2} \mathrm{~d} s\right\}<\infty .
$$

Definition 2. If the CVNNs satisfy Definition 1 under the initial condition, and $\|G\|_{\infty}<\gamma$, that is,

$$
\int_{0}^{\infty} v^{H}(s) v(s) \mathrm{d} s \leq \gamma^{2} \int_{0}^{\infty} \omega^{H}(s) \omega(s) \mathrm{d} s,
$$

then we say system (5) possesses $H \infty$ performance with attenuation index $\gamma$.

Several essential lemmas are given before the proof of our theorems.

Lemma 1 (see [12]). For any vector $\zeta \in \mathscr{C}^{m}$, there is a scalar $\gamma \in(0,1)$, and $\mathscr{H} \in \mathscr{C}^{n \times n}$ is a positive defined matrix, matrices $F_{1}, F_{2} \in \mathscr{C}^{n \times m}$, and $\Phi(\gamma, \mathscr{H})$ is considered as

$$
\Phi(\gamma, \mathscr{H})=\frac{1}{\gamma} \zeta^{H} F_{1}^{H} \mathscr{H} F_{1} \zeta+\frac{1}{1-\gamma} \zeta^{H} F_{2}^{H} S F_{2} \zeta .
$$

If the matrix $U \in \mathscr{C}^{n \times n}$ satisfies $\left[\begin{array}{cc}\mathscr{H} & U \\ * & \mathscr{H}\end{array}\right]>0$, then

$$
\min _{\beta \in(0,1)} \Phi(\gamma, \mathscr{H}) \geq\left[\begin{array}{l}
F_{1} \zeta \\
F_{2} \zeta
\end{array}\right]^{H}\left[\begin{array}{ll}
\mathscr{H} & U \\
* & \mathscr{H}
\end{array}\right]\left[\begin{array}{l}
F_{1} \zeta \\
F_{2} \zeta
\end{array}\right] \text {. }
$$

Lemma 2 (see [41, 42]). $\mathfrak{R}>0$ is a Hermitian matrix, for any continuous and differentiable function $\vartheta:[c, d] \longrightarrow \mathscr{C}^{n}$; the following inequality holds

$$
\int_{c}^{d} \vartheta^{H}(s) \Re \vartheta(s) \mathrm{d} s \geq \frac{\Theta_{1}^{H} \mathfrak{R} \Theta_{1}+3 \Theta_{2}^{H} \mathfrak{R} \Theta_{2}}{d-c},
$$

where

$$
\begin{aligned}
& \Theta_{1}=\vartheta(d)-\vartheta(c), \\
& \Theta_{2}=\vartheta(d)+\vartheta(c)-\frac{2}{d-c} \int_{c}^{d} \vartheta(s) \mathrm{d} s .
\end{aligned}
$$

By combining Lemma 1 with Lemma 2, we have the following lemma.
Lemma 3. For Hermitian matrix $\mathfrak{\mathbb { S }}$ and arbitrary matrices $\mathcal{S}_{11}, \mathcal{S}_{22}, \mathcal{S}_{12}$, which belong to $\mathscr{C}^{n \times n}$, setting $0<\sigma(t) \leq \sigma$, we have

$$
-\sigma \int_{t-\sigma}^{t} \dot{\vartheta}^{H}(s) \widetilde{S} \dot{\vartheta}(s) \mathrm{d} s \leq-\Gamma^{H} \Omega \Gamma
$$

where

$$
\Omega=\left[\begin{array}{cccc}
\mathfrak{S} & 0 & \mathcal{S}_{11} & \mathcal{S}_{12} \\
* & 3 \mathfrak{S} & \mathcal{S}_{12}^{H} & \mathcal{S}_{22} \\
* & * & \mathfrak{S} & 0 \\
* & * & * & 3 \mathfrak{S}
\end{array}\right] \geq 0,
$$

$$
\Gamma=\left[\begin{array}{c}
\vartheta(t)-\vartheta(t-\sigma(t)) \\
\vartheta(t)+\vartheta(t-\sigma(t))-\frac{2}{\sigma(t)} \int_{t-\sigma(t)}^{t} \vartheta(s) \mathrm{d} s \\
\vartheta(t-\sigma(t))-\vartheta(t-\sigma) \\
\vartheta(t-\sigma(t))+\vartheta(t-\sigma)-\frac{2}{\sigma-\sigma(t)} \int_{t-\sigma}^{t-\sigma(t)} \vartheta(s) \mathrm{d} s
\end{array}\right] .
$$

Proof. By dividing the left-hand side of inequality (18) into two parts, the following formula is obtained:

$$
\begin{aligned}
-\sigma \int_{t-\sigma}^{t} \dot{\vartheta}^{H}(s) \mathfrak{S} \dot{\vartheta}(s) \mathrm{d} s= & -\sigma \int_{t-\sigma(t)}^{t} \dot{\vartheta}^{H}(s) \\
& \cdot \mathfrak{S} \dot{\mathfrak{\vartheta}}(s) \mathrm{d} s-\sigma \int_{t-\sigma}^{t-\sigma(t)} \dot{\mathfrak{\vartheta}}^{H}(s) \mathfrak{S} \dot{\mathfrak{\vartheta}}(s) \mathrm{d} s,
\end{aligned}
$$

estimating the two parts of the right-hand side of the above inequality, respectively, by Lemma 2, and then, combining the obtained parts via Lemma 1, we have

$$
\begin{aligned}
-\sigma \int_{t-\sigma}^{t} \dot{\vartheta}^{H}(s) \mathfrak{\Im} \dot{\vartheta}(s) \mathrm{d} s & \leq-\frac{\sigma\left(\eta_{1}^{H} \mathfrak{s} \eta_{1}+3 \eta_{2}^{H} \mathfrak{S} \eta_{2}\right)}{\sigma(t)}-\frac{\sigma\left(\eta_{3}^{H} \mathfrak{S} \eta_{3}+3 \eta_{4}^{H} \mathfrak{s} \eta_{4}\right)}{\sigma-\sigma(t)} \\
& \leq-\left[\begin{array}{l}
\eta_{1} \\
\eta_{2} \\
\eta_{3} \\
\eta_{4}
\end{array}\right]\left[\begin{array}{cccc}
\mathfrak{s} & 0 & \mathcal{S}_{11} & \mathcal{S}_{12} \\
* & 3 \mathfrak{s} & \mathcal{S}_{12}^{H} & \mathcal{S}_{22} \\
* & * & \mathfrak{S} & 0 \\
* & * & * & 3 \mathfrak{S}
\end{array}\right]\left[\begin{array}{l}
\eta_{1} \\
\eta_{2} \\
\eta_{3} \\
\eta_{4}
\end{array}\right]
\end{aligned}
$$

with 


$$
\begin{aligned}
& \eta_{1}^{H}=\vartheta^{H}(t)-\vartheta^{H}(t-\sigma(t)), \\
& \eta_{2}^{H}=\vartheta^{H}(t)+\vartheta^{H}(t-\sigma(t))-\frac{2}{\sigma(t)} \int_{t-\sigma(t)}^{t} \vartheta^{H}(s) \mathrm{d} s \\
& \eta_{3}^{H}=\vartheta^{H}(t-\sigma(t))-\vartheta^{H}(t-\sigma), \\
& \eta_{4}^{H}=\vartheta^{H}(t-\sigma(t))+\vartheta^{H}(t-\sigma)-\frac{2}{\sigma-\sigma(t)} \int_{t-\sigma}^{t-\sigma(t)} \vartheta^{H}(s) \mathrm{d} s,
\end{aligned}
$$

Remark 2. A similar conclusion has been obtained in real domain. Lemma 3 is an extension which can apply to the complex domain with complex-valued matrices and vectors $\vartheta(t), \vartheta(t-\sigma(t))$. It is worth noting that the quadratic form $\dot{\vartheta}^{H}(s) \mathfrak{S} \dot{\mathcal{\vartheta}}(s)$ is in the real number field because $\mathbb{S}$ is a Hermitian matrix.

Lemma 4 (see [35]). Set $U>0$ as an arbitrary matrix in $R^{n}$, and $v(t)$ is a vector with appropriate dimensional. Thus, the following inequality is obtained:

and this ends the proof.

$$
-\int_{t_{1}}^{t_{2}} v^{T}(s) U v(s) \mathrm{d} s \leq\left(t_{2}-t_{1}\right) \lambda^{T}(t) V^{T} U^{-1} V \lambda(t)+2 \lambda^{T}(t) V^{T} \int_{t_{1}}^{t_{2}} v(s) \mathrm{d} s
$$

for any $V$ and vector $\lambda(t)$ with appropriate dimensional are not dependent on integral variables.

This lemma is currently only used in the real number field; the following corollary will extend it to the domain of complex numbers.

Corollary 1. $\mathrm{U}$ is a positive defined Hermitian matrix, and $\xi(t)$ is a complex vector with appropriate dimensional. Thus, the following inequality is obtained:

$$
\begin{gathered}
-\int_{t_{1}}^{t_{2}} \xi^{H}(s) \mathrm{U} \xi(s) \mathrm{d} s \leq\left(t_{2}-t_{1}\right) \lambda^{H}(t) V^{H} \mathrm{U}^{-1} V \lambda(t) \\
+\lambda^{H}(t) V^{H} \int_{t_{1}}^{t_{2}} \xi(s) \mathrm{d} s+\int_{t_{1}}^{t_{2}} \xi(s) \mathrm{d} s V \lambda(t),
\end{gathered}
$$

for any $V$ and vector $\lambda(t)$ with appropriate dimensional are not dependent on integral variables.

Proof. $\mathrm{U}>0$ is a Hermitian matrix; for complex vectors $a, b$ with appropriate dimension, we have

$$
(a+Y b)^{H} Y^{-1}(a+Y b) \geq 0,
$$

that is to say,

$$
a^{H} Y^{-1} a+a^{H} b+b^{H} a+b^{H} Y b \geq 0,
$$

and, then, the following conclusion is obtained:

$$
\begin{aligned}
- & \lambda^{H}(t) V^{H} \int_{t_{1}}^{t_{2}} \xi(s) \mathrm{d} s-\int_{t_{1}}^{t_{2}} \xi^{H}(s) \mathrm{d} s V \lambda(t) \\
& =\int_{t_{1}}^{t_{2}}\left[-\lambda^{H}(t) V^{H} \xi(s)-\xi^{H}(s) V \lambda t\right] \mathrm{d} s \\
& \leq \int_{t_{1}}^{t_{2}} \lambda^{H}(t) V^{H} \mathrm{U}^{-1} V \lambda(t) \mathrm{d} s+\int_{t_{1}}^{t_{2}} \xi^{H}(s) \mathrm{U} \xi(s) \mathrm{d} s \\
& =\left(t_{2}-t_{1}\right) \lambda^{H}(t) V^{H} \mathrm{U}^{-1} V \lambda(t)+\int_{t_{1}}^{t_{2}} \xi^{H}(s) \mathrm{U} \xi(s) \mathrm{d} s .
\end{aligned}
$$

This ends the proof.

Remark 3. In the proof of Corollary 1 , we can clearly see that $-\lambda^{H}(t) V^{H} \int_{t_{1}}^{t_{2}} \xi(s) \mathrm{d} s$ and $-\int_{t_{1}}^{t_{2}} \xi(s) \mathrm{d} s V \lambda(t)$ cannot be merged into $-2 \lambda^{H}(t) V^{H} \int_{t_{1}}^{t_{2}} \xi(s) \mathrm{d} s$ because it may be an imaginary number. Also, the condition $U>0$ in Lemma 4 is changed into $U$, which is a positive defined Hermitian matrix, which can ensure that both sides of the inequality are real numbers so that the magnitudes can be compared. Therefore, the above inequality can be used in the complex domain in the form of Corollary 1.

\section{Main Result}

Two main theorems will be presented in this section. In the first place, we study the stabilization of system (5) with $H \infty$ performance without a SBET scheme; and then, by 
employing the SBET scheme (6), we derive a sufficient condition for the stability of system (8). Now we define the following vectors and matrices for clarity:

$$
\begin{aligned}
& v_{1}=\frac{1}{\sigma(t)} \int_{t-\sigma(t)}^{t} 9(s) \mathrm{d} s, \\
& v_{2}=\frac{1}{\sigma-\sigma(t)} \int_{t-\sigma}^{t-\sigma(t)} 9(s) \mathrm{d} s, \\
& v_{3}=\frac{1}{\eta(t)} \int_{t-\eta(t)}^{t} 9(s) \mathrm{d} s, \\
& v_{4}=\frac{1}{\eta-\eta(t)} \int_{t-\eta}^{t-\eta(t)} 9(s) \mathrm{d} s, \\
& v_{5}=\frac{1}{\eta *(t)} \int_{t-\eta^{*}(t)}^{t} 9(s) \mathrm{d} s, \\
& v_{6}=\frac{1}{\eta-\eta *(t)} \int_{t-\eta}^{t-\eta^{*}(t)} 9(s) \mathrm{d} s, \\
& \overline{Q_{1}}=\left[\begin{array}{cccc}
\widehat{Q}_{3} & 0 & \hat{\mathcal{S}}_{11} & \hat{\mathcal{S}}_{12} \\
* & 3 \widehat{Q}_{3} & \hat{\mathcal{S}}_{12}^{H} & \hat{\mathcal{S}}_{22} \\
* & * & \widehat{Q}_{3} & 0 \\
* & * & * & 3 \widehat{Q}_{3}
\end{array}\right] \geq 0, \\
& \overline{\widehat{Q}_{2}}=\left[\begin{array}{cccc}
\widehat{Q}_{4} & 0 & \hat{\mathcal{S}}_{33} & \hat{\mathcal{S}}_{34} \\
* & 3 \widehat{Q}_{4} & \hat{\mathcal{S}}_{34}^{H} & \hat{\mathcal{S}}_{44} \\
* & * & \widehat{Q}_{4} & 0 \\
* & * & * & 3 \widehat{Q}_{4}
\end{array}\right] \geq 0, \\
& \overline{\widehat{Q}_{3}}=\left[\begin{array}{cccc}
\widehat{Q}_{6} & 0 & \hat{\delta}_{55} & \hat{\delta}_{56} \\
* & 3 \widehat{Q}_{6} & \hat{\mathcal{S}}_{56}^{H} & \hat{\mathcal{S}}_{66} \\
* & * & \widehat{Q}_{6} & 0 \\
* & * & * & 3 \widehat{Q}_{6}
\end{array}\right] \geq 0, \\
& Q_{1}=\left[\begin{array}{cccc}
Q_{3} & 0 & \mathcal{S}_{11} & \mathcal{S}_{12} \\
* & 3 Q_{3} & \mathcal{S}_{12}^{H} & \mathcal{S}_{22} \\
* & * & Q_{3} & 0 \\
* & * & * & 3 Q_{3}
\end{array}\right] \geq 0, \\
& Q_{2}=\left[\begin{array}{cccc}
Q_{4} & 0 & \mathcal{S}_{33} & \mathcal{S}_{34} \\
* & 3 \mathscr{Q}_{4} & \mathcal{S}_{34}^{H} & \mathcal{S}_{44} \\
* & * & \mathbb{Q}_{4} & 0 \\
* & * & * & 3 \mathscr{Q}_{4}
\end{array}\right] \geq 0, \\
& Q_{3}=\left[\begin{array}{cccc}
Q_{6} & 0 & \mathcal{S}_{55} & \mathcal{S}_{56} \\
* & 3 Q_{6} & \mathcal{S}_{56}^{H} & \mathcal{S}_{66} \\
* & * & Q_{6} & 0 \\
* & * & * & 3 Q_{6}
\end{array}\right] \geq 0 .
\end{aligned}
$$

Theorem 1. Under the condition of Assumption 1, for given scalar $\sigma, \sigma_{d}$ and $m$, if there exist positive defined Hermitian matrices $\widehat{\mathscr{P}}_{p}, \widehat{\mathscr{Q}}_{p}, \widehat{\mathscr{Q}}_{3}, \widehat{\mathscr{W}}_{p}$ in $\mathscr{C}^{n \times n}, \widehat{\mathscr{R}}_{p}, \widehat{\mathscr{R}}, \widehat{\mathscr{M}}_{p} \in \mathscr{C}^{2 n \times 2 n}$, $\widehat{E}_{1}, \widehat{E}_{2}$ are positive diagonal matrices, and any matrices $Y_{1 p}, \widehat{S}_{11}, \widehat{\mathcal{S}}_{12}, \widehat{\mathcal{S}}_{22}, \mathscr{K}_{p}$, nonsingular matrix $\mathscr{X}$ with proper dimension such that the following LMIs, are established with $p \in\{1,2,3\}$ :

$$
\begin{aligned}
& \Omega_{1}=\left[\begin{array}{cc}
\Xi_{1}+\Xi_{2} & \Lambda \\
* & -I
\end{array}\right]<0, \\
& \sum_{q \in \mathbb{N}_{k}^{p}}^{n} \pi_{p q}\left(\widehat{\mathscr{R}}_{q}-\widehat{\mathscr{M}}_{p}\right) \leq \widehat{\mathscr{R}}, \\
& \widehat{\mathscr{P}}_{q}-\widehat{\mathscr{W}}_{p} \leq 0, q \in \aleph_{u k}^{p}, p \neq q, \widehat{\mathscr{R}}_{q}-\widehat{\mathscr{M}}_{p} \leq 0, \\
& q \in \aleph_{u k}^{p}, p \neq q, \\
& \widehat{\mathscr{P}}_{q}-\widehat{\mathscr{W}}_{p} \geq 0, q \in \aleph_{u k}^{p}, p=q, \widehat{\mathscr{R}}_{q}-\widehat{\mathscr{M}}_{p} \geq 0, \\
& q \in \aleph_{u k}^{p}, p=q,
\end{aligned}
$$

where

$$
\begin{aligned}
& \Xi_{1}=e_{10}^{H} \widehat{Q}_{1} e_{10}-\left(1-\sigma_{d}\right) e_{8}^{H} \widehat{Q}_{1} e_{8}+e_{1}^{H} \widehat{Q}_{2} e_{1}-\left(1-\sigma_{d}\right) \\
& \cdot e_{3}^{H} \widehat{Q}_{2} e_{3}+\sigma^{2} e_{10}^{H} \widehat{Q}_{3} e_{10}-\Gamma_{1}^{H} \widehat{Q}_{1} \Gamma_{1}+\sigma \Gamma_{2}^{H} \widehat{\mathscr{R}}_{2} \\
& +\operatorname{sym}\left\{e_{1}^{H} \widehat{\mathscr{P}}_{p} e_{10}\right\}+\Gamma_{2}^{H} \widehat{\mathscr{R}}_{p} \Gamma_{2}+\Gamma_{3}^{H} \widehat{\mathscr{R}}_{p} \Gamma_{3} \\
& +e_{1}^{H} \sum_{q \in \aleph_{k}^{p}}^{n} \pi_{p q}\left(\widehat{\mathscr{P}}_{q}-\widehat{\mathscr{W}}_{p}\right) e_{1}+e_{1}^{H} L^{H} \widehat{E}_{1} L e_{1}-e_{4}^{H} \widehat{E}_{1} e_{4} \\
& +e_{3}^{H} L^{H} \widehat{E}_{2} L e_{3}-e_{5}^{H} \widehat{E}_{2} e_{5}-\gamma^{2} e_{11}^{H} \widehat{I} e_{11}-e_{12}^{H} \widehat{I} e_{12}, \\
& \Xi_{2}=\operatorname{sym}\left\{( m e _ { 1 } ^ { H } + e _ { 1 0 } ^ { H } ) \left(-\mathscr{X} e_{10}-\left(\mathscr{A}_{1 p} \mathscr{X}-\mathrm{E}_{2 p} Y_{p}\right) e_{1}\right.\right. \\
& \left.\left.+\mathscr{B}_{1 p} \mathscr{X} e_{8}+\mathscr{C}_{1 p} \mathscr{X} e_{4}+\mathscr{D}_{1 p} \mathscr{X} e_{5}+\mathscr{E}_{1 p} \mathscr{X} e_{11}\right)\right\}, \\
& \Lambda=\left[-\mathscr{X}_{2 p}, 0,0, \mathscr{X}_{2 p}, \mathscr{X}_{2 p}, 0,0,0, \mathscr{X}_{1 p}\right] \text {, } \\
& \xi_{1}^{H}(t)=\left[\vartheta^{H}(t), \vartheta^{H}(t-\sigma), \vartheta^{H}(t-\sigma(t)), \varphi^{H}(\vartheta(t)),\right. \\
& \varphi^{H}(\vartheta(t-\sigma(t))), v_{1}^{H}, v_{2}^{H}, \dot{\vartheta}^{H}(t-\sigma(t)), \\
& \varphi^{H}(\vartheta(t-\sigma), \dot{\vartheta}(t), \omega(t)]^{H}, \\
& \xi_{2}^{H}(t)=\left[\vartheta^{H}(t), \varphi^{H}(\vartheta(t))\right]^{H}, \\
& e_{p}=\left[\begin{array}{lll}
0_{n \times(p-1) n} & I_{n} & 0_{n \times(11-p) n}
\end{array}\right], \\
& \Gamma_{1}=\left[\begin{array}{c}
e_{1}-e_{3} \\
e_{1}+e_{3}-2 e_{6} \\
e_{3}-e_{2} \\
e_{3}+e_{2}-2 e_{7}
\end{array}\right] \text {, } \\
& \Gamma_{2}=\left[e_{1}, e_{4}\right] \text {, } \\
& \Gamma_{3}=\left[e_{2}, e_{9}\right] \text {. }
\end{aligned}
$$


Then, system (5) is asymptotically stable via $u(t)=\mathscr{K}_{p} \vartheta(t)$ with Ho disturbance attenuation $\gamma$ and the controller gain matrices are designed as $\mathscr{K}_{p}=Y_{1 p} \mathscr{X}^{-1}$.

Proof. Consider the following LKF:

$$
V(\vartheta(t), t, p)=\sum_{m=1}^{5} V_{m}(\vartheta(t), t, p)
$$

where

$$
\begin{aligned}
& V_{1}(\vartheta(t), p)=\vartheta^{H}(t) \mathscr{P}_{p} \vartheta(t), \\
& V_{2}(\vartheta(t), p)=\int_{t-\sigma}^{t} \xi_{2}^{H}(s) \mathscr{R}_{p} \xi_{2}(s) \mathrm{d} s, \\
& V_{3}(\vartheta(t), p)=\int_{t-\sigma(t)}^{t} \dot{\vartheta}^{H}(s) \mathscr{Q}_{1} \dot{\vartheta}(s) \mathrm{d} s+\int_{t-\sigma(t)}^{t} \vartheta^{H}(s) \mathscr{Q}_{2} \vartheta(s) \mathrm{d} s, \\
& V_{4}(\vartheta(t), p)=\sigma \int_{\sigma}^{0} \int_{t-\theta}^{t} \dot{\vartheta}^{H}(s) \mathscr{Q}_{3} \dot{\vartheta}(s) \mathrm{d} s \mathrm{~d} \theta \\
& V_{5}(\vartheta(t), p)=\int_{t-\sigma}^{t} \int_{\theta}^{0} \xi_{2}^{H}(s) \mathscr{R} \xi_{2}(s) \mathrm{d} s \mathrm{~d} \theta
\end{aligned}
$$

with

$$
\mathscr{P}_{p}=\left(X^{H}\right)^{-1} \widehat{\mathscr{P}}_{p} \mathscr{X}^{-1}, \widehat{Q}_{p}=\left(X^{H}\right)^{-1} \widehat{Q}_{p} \mathscr{X}^{-1}, \mathscr{R}_{p}=\left(X^{H}\right)^{-1} \widehat{\mathscr{R}}_{p} \mathscr{X}^{-1}, \mathscr{R}=\left(X^{H}\right)^{-1} \widehat{\mathscr{R}} \mathscr{X}^{-1}, \quad p \in\{1,2,3\} .
$$

Adopting the weak infinitesimal operator $L$ in [21], which acts on $V_{m}(\vartheta(t), t, i)$ as

$$
L V(\vartheta(t), p)=\lim _{\Delta \longrightarrow \infty} \frac{1}{\Delta}\left[\mathbb{E}\left\{V\left(\vartheta(t+\Delta), t, r_{t+\Delta}\right) \mid \vartheta(t), r_{t}=p\right\}-V(\vartheta(t), t, p)\right]
$$

then, similar to the computation in $[26,27]$, we have

$$
\begin{aligned}
& L V_{1}(\vartheta(t), p)=\vartheta^{H}(t) \mathscr{P}_{p} \dot{\vartheta}(t)+\dot{\vartheta}^{H}(t) \mathscr{P}_{p} \vartheta(t)+\vartheta^{H}(t) \sum_{q=1}^{n} \pi_{p q} \mathscr{P}_{q} \vartheta(t) \\
& L V_{2}(\vartheta(t), p)=\xi_{2}^{H}(t) \mathscr{R}_{p} \xi_{2}(t)-\xi_{2}^{H}(t-\sigma) \mathscr{R}_{p} \xi_{2}(t-\sigma)+\int_{t-\sigma}^{t} \xi_{2}^{H}(s) \sum_{q=1}^{n} \pi_{p q} \mathscr{R}_{q} \xi_{2}(s) \mathrm{d} s,
\end{aligned}
$$

and considering the transition rate matrix includes not only the known part but also the unknown part, the following equations would hold for matrices $\mathscr{W}_{p}=\left(\mathscr{X}^{H}\right)^{-1}$

$$
\begin{aligned}
& 0=-\vartheta^{H}(t)\left(\sum_{q=1}^{n} \pi_{p q} \mathscr{W}_{p}\right) \vartheta(t), \quad \forall p \in \wp, \\
& 0=-\int_{t-\sigma}^{t} \vartheta^{H}(s)\left(\sum_{q=1}^{n} \pi_{p q} \mathscr{M}_{p}\right) \vartheta(s) \mathrm{d} s, \quad \forall p \in \wp .
\end{aligned}
$$


Then, (36) and (37) are equal to the following equations:

$$
\begin{aligned}
& L V_{1}(\vartheta(t), p)=\vartheta^{H}(t) \mathscr{P}_{p} \dot{\vartheta}(t)+\dot{\vartheta}^{H}(t) \mathscr{P}_{p} \vartheta(t)+\vartheta^{H}(t) \sum_{q \in \aleph_{k}^{p}} \pi_{p q}\left(\mathscr{P}_{q}-\mathscr{W}_{p}\right) \vartheta(t)+\vartheta^{H}(t) \\
& \quad \cdot \sum_{q \in \aleph_{u k}^{p}} \pi_{p q}\left(\mathscr{P}_{q}-\mathscr{W}_{p}\right) \vartheta(t), \\
& L V_{2}(\vartheta(t), p)=\xi_{2}^{H}(t) \mathscr{R}_{p} \xi_{2}(t)-\xi_{2}^{H}(t-\sigma) \mathscr{R}_{p} \xi_{2}(t-\sigma)+\int_{t-\sigma}^{t} \xi_{2}^{H}(s) \sum_{q \in \aleph_{k}^{p}} \pi_{p q}\left(\mathscr{R}_{q}-\mathscr{M}_{p}\right) \xi(s)_{2} \mathrm{~d} s \\
& +\int_{t-\sigma}^{t} \xi_{2}^{H}(s) \sum_{q \in \aleph_{u k}^{p}} \pi_{p q}\left(R_{q}-\mathscr{M}_{p}\right) \xi_{2}(s) \mathrm{ds} .
\end{aligned}
$$

Via easy calculations, the following equations are given:

$$
\begin{aligned}
L V_{3}(\vartheta(t), p) \leq & \dot{\vartheta}^{H}(t) \mathscr{Q}_{1} \dot{\vartheta}(t)-\left(1-\sigma_{d}\right) \dot{\vartheta}^{H}(t-\sigma(t)) Q_{1} \dot{\vartheta}(t-\sigma(t)) \\
& +\vartheta^{H}(t) \mathscr{Q}_{2} \vartheta(t)-\left(1-\sigma_{d}\right) \vartheta^{H}(t-\sigma(t)) Q_{2} \vartheta(t-\sigma(t)), \\
L V_{4}(\vartheta(t), p)= & \sigma^{2} \dot{\vartheta}^{H}(t) \mathscr{Q}_{3} \dot{\vartheta}(t)-\sigma \int_{t-\sigma}^{t} \dot{\vartheta}^{H}(s) \mathscr{Q}_{3} \dot{\vartheta}(s) \mathrm{d} s \\
L V_{5}(\vartheta(t), p)= & \sigma \xi_{2}^{H}(t) \mathscr{R} \xi_{2}(t)-\int_{t-\sigma}^{t} \xi_{2}^{H}(s) \mathscr{R} \xi_{2}(s) \mathrm{d} s .
\end{aligned}
$$

Dealing with the integral term containing the Markov jump, by pre- and postmultiplying $\left(\mathscr{X}^{H}\right)^{-1}$ and $\mathscr{X}^{-1}$ with (30), we have

$$
\begin{aligned}
& \sum_{q \in \aleph_{k}^{p}}^{n} \pi_{p q}\left(\mathscr{R}_{q}-\mathscr{M}_{p}\right) \leq \mathscr{R}, \\
& \mathscr{P}_{q}-\mathscr{W}_{p} \leq 0, q \in \aleph_{u k}^{p}, p \neq q, \mathscr{R}_{q}-\mathscr{M}_{p} \leq 0, q \in \aleph_{u k}^{p}, p \neq q, \\
& \mathscr{P}_{q}-\mathscr{W}_{p} \geq 0, q \in \aleph_{u k}^{p}, p=q, \mathscr{R}_{q}-\mathscr{M}_{p} \geq 0, q \in \aleph_{u k}^{p}, p=q,
\end{aligned}
$$

and then, the following inequalities are obtained:

$$
\begin{aligned}
& \int_{t-\sigma}^{t} \xi_{2}^{H}(s) \sum_{q \in \aleph_{k}^{p}} \pi_{p q}\left(\mathscr{R}_{q}-\mathscr{M}_{p}\right) \xi_{2}(s) \mathrm{d} s-\int_{t-\sigma}^{t} \xi_{2}^{H}(s) \mathscr{R} \xi_{2}(s) \mathrm{d} s \leq 0, \\
& \vartheta^{H}(t) \sum_{q \in \aleph_{u k}^{p}} \pi_{p q}\left(\mathscr{P}_{q}-\mathscr{W}_{p}\right) \vartheta(t)+\int_{t-\sigma}^{t} \xi_{2}^{H}(s) \sum_{q \in \aleph_{u k}^{p}} \pi_{p q}\left(\mathscr{R}_{q}-\mathscr{M}_{p}\right) \xi_{2}(s) \mathrm{d} s \leq 0 .
\end{aligned}
$$


For arbitrary matrices $\mathcal{S}_{11}, \mathcal{S}_{22}, \mathcal{\delta}_{12} \in \mathscr{C}^{n \times n}$, because of Lemma $3,-\sigma \int_{t-\sigma}^{t} \dot{\vartheta}^{H}(s) Q_{3} \dot{\vartheta}(s) \mathrm{d} s$ in (42) will be processed as follows: by dividing it into two parts, we have

$$
-\sigma \int_{t-\sigma}^{t} \dot{\vartheta}^{H}(s) Q_{3} \dot{\vartheta}(s) \mathrm{d} s=-\sigma \int_{t-\sigma(t)}^{t} \dot{\vartheta}^{H}(s) Q_{3} \dot{\vartheta}(s) \mathrm{d} s-\sigma \int_{t-\sigma}^{t-\sigma(t)} \dot{\vartheta}^{H}(s) Q_{3} \dot{\vartheta}(s) \mathrm{d} s,
$$

estimating the two parts of the right-hand side of the above inequality, respectively, by Lemma 2 , and then, combining the obtained parts via Lemma 1, we have

$$
\begin{aligned}
-\sigma \int_{t-\sigma}^{t} \dot{\vartheta}^{H}(s) Q_{3} \dot{\vartheta}(s) \mathrm{d} s & \leq-\frac{\sigma\left(\eta_{1}^{H} Q_{3} \eta_{1}+3 \eta_{2}^{H} Q_{3} \eta_{2}\right)}{\sigma(t)}-\frac{\sigma\left(\eta_{3}^{H} Q_{3} \eta_{3}+3 \eta_{4}^{H} Q_{3} \eta_{4}\right)}{\sigma-\sigma(t)} \\
& \leq-\left[\begin{array}{l}
\eta_{1} \\
\eta_{2} \\
\eta_{3} \\
\eta_{4}
\end{array}\right]\left[\begin{array}{cccc}
Q_{3} & 0 & \mathcal{S}_{11} & \mathcal{S}_{12} \\
* & 3 Q_{3} & \mathcal{S}_{12}^{H} & \mathcal{S}_{22} \\
* & * & \mathcal{Q}_{3} & 0 \\
* & * & * & 3 Q_{3}
\end{array}\right]\left[\begin{array}{l}
\eta_{1} \\
\eta_{2} \\
\eta_{3} \\
\eta_{4}
\end{array}\right] \\
& =-\xi_{1}^{H}(t) \Gamma_{1}^{H} Q_{1} \Gamma_{1} \xi_{1}(t) .
\end{aligned}
$$

Thus,

$$
L V_{4}(\vartheta(t), p) \leq \sigma^{2} \dot{\vartheta}^{H}(t) \mathcal{Q}_{3} \dot{\vartheta}(t)-\xi_{1}^{H}(t) \Gamma_{1}^{H} Q_{1} \Gamma_{1} \xi_{1}(t) .
$$

On the other hand, for diagonal matrices $E_{1}=\left(\mathscr{X}^{H}\right)^{-1} \widehat{E}_{1} \mathscr{X}^{-1}, E_{2}=\left(\mathscr{X}^{H}\right)^{-1} \widehat{E}_{2} \mathscr{X}^{-1}$, Assumption 1 implies that

$$
\begin{aligned}
& \vartheta^{H}(t) L E_{1} L \vartheta(t)-\varphi^{H}(\vartheta(t)) E_{1} \varphi(\vartheta(t)) \geq 0 \\
& \vartheta^{H}(t-\sigma(t)) L E_{2} L \vartheta(t-\sigma(t))-\varphi^{H}(\vartheta(t-\sigma(t))) E_{2} \varphi(\vartheta(t-\sigma(t))) \geq 0 .
\end{aligned}
$$

One can also obtain the following equation from (5) for any nonsingular matrix $\mathscr{X}_{2}$ with the appropriate dimension, we defined $\mathscr{X}_{1}=m \mathscr{X}_{2}$, and it is clearly that $\mathscr{X}_{2}$ is also nonsingular, such that

$$
\begin{aligned}
0 & =\left(\vartheta^{H}(t) \mathscr{X}_{1}^{H}+\dot{\vartheta}^{H}(t) \mathscr{X}_{2}^{H}\right)\left(-\dot{\vartheta}(t)+\left(-\mathscr{A}_{1 p}+\mathrm{E}_{2 p} \mathscr{K}_{p}\right) \vartheta(t)+\mathscr{B}_{1 p} \dot{\vartheta}(t-\sigma(t))+\mathscr{C}_{1 p} \varphi(\vartheta(t))+\mathscr{D}_{1 p} \varphi(\vartheta(t-\sigma(t)))\right. \\
& \left.+\mathscr{E}_{1 p} \omega(t)\right)+\left(-\dot{\vartheta}(t)+\left(-\mathscr{A}_{1 p}+\mathscr{E}_{2 p} \mathscr{K}_{p}\right) \vartheta(t)+\mathscr{B}_{1 p} \dot{\vartheta}(t-\sigma(t))+\mathscr{C}_{1 p} \varphi(\vartheta(t))+\mathscr{D}_{1 p} \varphi(\vartheta(t-\sigma(t)))\right. \\
& \left.+\mathscr{E}_{1 p} \omega(t)\right)^{H}\left(\mathscr{X}_{1} \vartheta(t)+\mathscr{X}_{2} \dot{\vartheta}(t)\right) .
\end{aligned}
$$

Through the analysis in (39)-(50), we have 


$$
\begin{aligned}
L V(\vartheta(t), p)+v^{H}(t) v(t)-\gamma^{2} \omega^{H}(t) \omega(t) \leq & \vartheta^{H}(t) \mathscr{P}_{p} \dot{\vartheta}(t)+\dot{\vartheta}^{H}(t) \mathscr{P}_{p} \vartheta(t)+\vartheta^{H}(t) \sum_{q \in \mathcal{\aleph}_{k}^{p}} \pi_{p q}\left(\mathscr{P}_{q}-\mathscr{W}_{p}\right) \vartheta(t) \\
& +\xi_{2}^{H}(t) \mathscr{R}_{p} \xi_{2}(t)-\xi_{2}^{H}(t-\sigma) \mathscr{R}_{p} \xi_{2}(t-\sigma)+\dot{\vartheta}^{H}(t)\left(\mathcal{Q}_{1}+\sigma^{2} \mathscr{Q}_{3}\right) \dot{\vartheta}(t) \\
& -\left(1-\sigma_{d}\right) \dot{\vartheta}^{H}(t-\sigma(t)) \mathscr{Q}_{1} \dot{\vartheta}(t-\sigma(t)) \\
& +\vartheta^{H}(t) \mathscr{Q}_{2} \vartheta(t)-\left(1-\sigma_{d}\right) \vartheta^{H}(t-\sigma(t)) \mathscr{Q}_{2} \vartheta(t-\sigma(t))-\xi_{1}^{H}(t) \Gamma_{1}^{H} \mathscr{Q}_{1} \Gamma_{1} \xi_{1}(t)+\sigma \xi_{2}^{H}(t) \mathscr{R} \xi_{2}(t) \\
& +\vartheta^{H}(t) L E_{1} L \vartheta(t) \\
& -\varphi^{H}(\vartheta(t)) E_{1} \varphi(\vartheta(t))+\vartheta^{H}(t-\sigma(t)) L E_{2} L \vartheta(t-\sigma(t))-\varphi^{H}(\vartheta(t-\sigma(t))) E_{2} \varphi(\vartheta(t-\sigma(t))) \\
& +\operatorname{sym}\left\{( \vartheta ^ { H } ( t ) \mathscr { X } _ { 1 } ^ { H } + \dot { \vartheta } ^ { H } ( t ) \mathscr { X } _ { 2 } ^ { H } ) \left(-\dot{\vartheta}(t)+\left(-\mathscr{A}_{1 p}+\mathscr{E}_{2 p} \mathscr{K}_{p}\right) \vartheta(t)+\mathscr{B}_{1 p} \dot{\vartheta}(t-\sigma(t))+\mathscr{C}_{1 p} \varphi(\vartheta(t))\right.\right. \\
& \left.\left.+\mathscr{D}_{1 p} \varphi(\vartheta(t-\sigma(t)))+\mathscr{E}_{1 p} \omega(t)\right)\right\}+v^{H}(t) v(t)-\gamma^{2} \omega^{H}(t) \omega(t)=\xi_{1}^{H} \Omega \xi_{1},
\end{aligned}
$$

where

$$
\begin{aligned}
\Omega= & \operatorname{sym}\left\{e_{1}^{H} \mathscr{P}_{p} e_{10}\right\}+e_{1}^{H} \sum_{q \in \aleph_{k}^{p}}^{n} \pi_{p q}\left(\mathscr{P}_{q}-\mathscr{W}_{p}\right) e_{1}+\Gamma_{2}^{H} \mathscr{R}_{p} \Gamma_{2}+\Gamma_{3}^{H} \mathscr{R}_{p} \Gamma_{3}+e_{10}^{H} Q_{1} e_{10}+\sigma^{2} e_{10}^{H} Q_{3} e_{10} \\
& -\left(1-\sigma_{d}\right) e_{8}^{H} Q_{1} e_{8}+e_{1}^{H} Q_{2} e_{1}-\left(1-\sigma_{d}\right) e_{3}^{H} Q_{2} e_{3}-\Gamma_{1}^{H} \mathscr{Q}_{1} \Gamma_{1}+\sigma \Gamma_{2}^{H} \mathscr{R}_{2}+e_{1}^{H} L^{H} E_{1} L e_{1}-e_{4}^{H} E_{1} e_{4} \\
& +\operatorname{sym}\left\{\left(e_{1}^{H} \mathscr{X}_{1}^{H}+e_{10}^{H} \mathscr{X}_{2}^{H}\right)\left(-e_{10}-\left(\mathscr{A}_{1 p}-\mathscr{E}_{2 p} \mathscr{K}_{p}\right) e_{1}+\mathscr{B}_{1 p} e_{8}+\mathscr{C}_{1 p} e_{4}+\mathscr{D}_{1 p} e_{5}+\mathscr{E}_{1 p} e_{11}\right)\right\} \\
& +e_{3}^{H} L^{H} E_{2} L e_{3}-e_{5}^{H} E_{2} e_{5}-\gamma^{2} e_{11}^{H} I e_{11}+\chi^{H} \chi \\
\chi= & -\mathscr{A}_{2 p} e_{1}+\mathscr{C}_{2 p} e_{4}+\mathscr{D}_{2 p} e_{5}+\mathscr{H}_{1 p} e_{11} .
\end{aligned}
$$

Defining

$$
\begin{aligned}
& \mathscr{X}=\mathscr{X}_{2}^{-1}, Y_{1 p}=\mathscr{K}_{p} \mathscr{X}, \mathfrak{X}_{\hbar}=\operatorname{diag}\{\underbrace{\mathscr{X}, \mathscr{X}, \ldots, \mathscr{X}}_{\hbar}\}, \widehat{\mathscr{P}}_{p}=\mathscr{X}^{H} \mathscr{P}_{p} \mathscr{X}, \widehat{\mathscr{W}}_{p}=\mathscr{X}^{H} \mathscr{W}_{p} \mathscr{X}, \\
& \widehat{\mathscr{M}}_{p}=\mathscr{X}^{H} \mathscr{M}_{p} \mathscr{X}, \widehat{\mathscr{P}}_{q}=\mathscr{X}^{H} \mathscr{P}_{q} \mathscr{X}, \widehat{\mathscr{R}}_{q}=\mathscr{X}^{H} \mathscr{R}_{q} \mathscr{X}, \widehat{\mathscr{R}}_{p}=\mathscr{X}^{H} \mathscr{R}_{p} \mathscr{X}, \widehat{Q}_{p}=\mathscr{X}^{H} Q_{p} \mathscr{X}, \widehat{Q}_{4}=\mathscr{X}^{H} \mathscr{Q}_{4} \mathscr{X}, \\
& \widehat{\mathscr{R}}=\mathscr{X}^{H} \mathrm{RX}, \hat{\mathscr{E}}_{1}=\mathscr{X}^{H} \mathscr{E}_{1} \mathscr{X}, \hat{\mathscr{E}}_{2}=\mathscr{X}^{H} \mathscr{E}_{2} \mathscr{X}, \widehat{I}=\mathscr{X}^{H} I X, \hat{\mathcal{S}}_{11}=\mathscr{X}^{H} \mathcal{S}_{11} \mathscr{X}, \hat{\mathcal{S}}_{12}=\mathscr{X}^{H} \mathcal{S}_{12} \mathscr{X}, \hat{\mathcal{S}}_{22}=\mathscr{X}^{H} \mathcal{S}_{22} \mathscr{X} \\
& L V(\vartheta(t), p)+v^{H}(t) v(t)-\gamma^{2} \omega^{H}(t) \omega(t)<0,
\end{aligned}
$$

and then, by pre- and postmultiplying $\mathfrak{X}_{\hbar}^{H}$ and $\mathfrak{X}_{\hbar}(\hbar=11)$ with $\Omega,(29)$ is obtained. Combining (29) with (51) and using the Schur complement, we have

and integrating the formula above from 0 to $\infty$, we have

$$
V(\vartheta(\infty))-V(\varphi(\theta)) \leq-\int_{0}^{\infty} v^{H}(t) v(t) \mathrm{d} t+\gamma^{2} \int_{0}^{\infty} \omega^{H}(t) \omega(t) \mathrm{d} t
$$

and, with the zero initial condition $\phi=0$, we have $V(\varphi(\theta))=0, V(\vartheta(\infty)) \geq 0$; thus,

$$
-\int_{0}^{\infty} v^{H}(t) v(t) \mathrm{d} t+\gamma^{2} \int_{0}^{\infty} \omega^{H}(t) \omega(t) \mathrm{d} t>V(\vartheta(\infty)) \geq 0 .
$$

This completes the proof.

Next, based on Theorem 1 and Lemma 3 and Corollary 1, a sampling-based event-triggered controller will be used to study the stabilization and Ho performance of CVNNs with uncertain Markov jump.

Theorem 2. For the given positive scalars $\sigma, \sigma_{d}, \rho, \eta$ and $0 \leq \omega<1$, if there exist Hermitian matrices $\widehat{\mathscr{P}}_{p}, \widehat{Q}_{p}, \widehat{Q}_{4}$, $\widehat{\mathscr{Q}}_{5}, \widehat{\mathscr{Q}}_{6}, \widehat{\mathscr{R}}, \widehat{\mathscr{G}}, \widehat{\mathscr{W}}_{p}$ in $\mathscr{C}^{n \times n}, \widehat{\mathscr{R}}_{p}, \widehat{\mathscr{R}}, \widehat{\mathscr{M}}_{p} \in \mathscr{C}^{2 n \times 2 n}$, and $E_{1}, E_{2}, \widehat{E}_{1}, \widehat{E}_{2}$ are positive diagonal matrices, matrices $\widetilde{\mathscr{K}}_{p}$, and nonsingular matrix $\widehat{\mathscr{X}} \in \mathscr{C}^{n * n}$ are proper dimension matrices with $p \in\{1,2,3\}$, such that the following conditions hold: 


$$
\begin{aligned}
& \widetilde{\Omega}_{2}(0)=\left[\begin{array}{cc}
\widehat{\Xi} & \widehat{\Lambda} \\
* & -I
\end{array}\right]<0, \\
& \widetilde{\Omega}_{2}(\eta)=\left[\begin{array}{cc}
\widehat{\Xi}+\eta \widehat{\Xi}_{1} & \widehat{\Lambda} \\
* & -I
\end{array}\right]<0,
\end{aligned}
$$

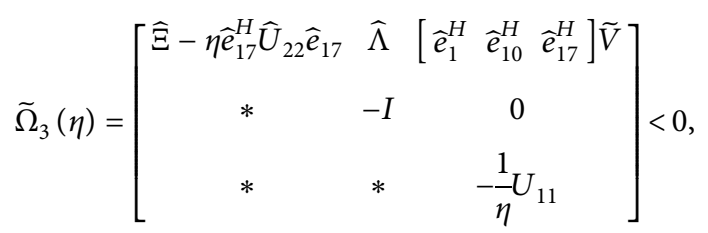

$$
\begin{aligned}
& \widehat{\mathscr{P}}_{q}-\widehat{\mathscr{W}}_{p} \leq 0, \quad q \in \aleph_{u k}^{p}, p \neq q, \\
& \widehat{\mathscr{R}}_{q}-\widehat{\mathscr{M}}_{p} \leq 0, \quad q \in \aleph_{u k}^{p}, p \neq q \text {, } \\
& \widehat{\mathscr{P}}_{q}-\widehat{\mathscr{W}}_{p} \geq 0, \quad q \in \aleph_{u k}^{p}, p=q \text {, } \\
& \widehat{\mathscr{R}}_{q}-\widehat{\mathscr{M}}_{p} \geq 0, \quad q \in \aleph_{u k}^{p}, p=q \text {, } \\
& \widehat{\Xi}=\operatorname{sym}\left\{\left(\rho \hat{e}_{1}^{H}+\hat{e}_{10}^{H}\right)\left(-\hat{\mathscr{X}} \hat{e}_{10}-\mathscr{A}_{1 p} \hat{\mathscr{X}} \hat{e}_{1}+\mathscr{B}_{1 p} \hat{\mathscr{X}} \hat{e}_{8}+\mathscr{C}_{1 p} \hat{\mathscr{X}} \hat{e}_{4}+\mathscr{D}_{1 p} \hat{\mathscr{X}} \hat{e}_{5}+\mathscr{E}_{1 p} \hat{\mathscr{X}} \hat{e}_{9}+\mathscr{E}_{2 p} Y_{2 p}\left(\hat{e}_{12}-\hat{e}_{14}\right)\right)\right\} \\
& +\widehat{e}_{10}^{H} \widehat{Q}_{1} \widehat{e}_{10}-\left(1-\sigma_{d}\right) \widehat{e}_{8}^{H} \widehat{Q}_{1} \widehat{e}_{8}+\widehat{e}_{1}^{H} \widehat{Q}_{2} \widehat{e}_{1}-\left(1-\sigma_{d}\right) \hat{e}_{3}^{H} \widehat{Q}_{2} \widehat{e}_{3}+\sigma^{2} \widehat{e}_{10}^{H} \widehat{Q}_{3} \widehat{e}_{10}-\Gamma_{1}^{H} \bar{Q}_{1} \Gamma_{1}+\sigma \Gamma_{2}^{H} \widehat{R} \Gamma_{2} \\
& +\operatorname{sym}\left\{\widehat{e}_{1}^{H} \widehat{\mathscr{P}}_{p} \widehat{e}_{10}\right\}+\Gamma_{2}^{H} \widehat{\mathscr{R}}_{p} \Gamma_{2}+\Gamma_{3}^{H} \widehat{\mathscr{R}}_{p} \Gamma_{3}+\widehat{e}_{1}^{H} \sum_{q \in \aleph_{k}^{p}}^{n} \pi_{p q}\left(\widehat{\mathscr{P}}_{q}-\widehat{\mathscr{W}}_{p}\right) \widehat{e}_{1}+\widehat{e}_{1}^{H} L^{H} \widehat{E}_{1} L \widehat{e}_{1}-\widehat{e}_{4}^{H} \widehat{E}_{1} \widehat{e}_{4} \\
& +\widehat{e}_{3}^{H} L^{H} \widehat{E}_{2} L \widehat{e}_{3}-\widehat{e}_{5}^{H} \widehat{E}_{2} \widehat{e}_{5}+\eta^{2} \widehat{e}_{10}^{H}\left(\widehat{Q}_{4}+\widehat{Q}_{6}\right) \widehat{e}_{10}-\Gamma_{4}^{H} \bar{Q}_{2} \Gamma_{4}-\Gamma_{5}^{H} \widehat{Q}_{3} \Gamma_{5}-\left(\widehat{e}_{1}-\widehat{e}_{17}\right)^{H} \widehat{Q}_{5}\left(\widehat{e}_{1}-\widehat{e}_{17}\right) \\
& -\Gamma_{7}^{H} \widehat{\mathscr{V}} \Gamma_{7}-\operatorname{sym}\left\{\left(\widehat{e}_{1}-\widehat{e}_{17}\right)^{H} \widehat{U}_{12} \widehat{e}_{17}\right\}+\operatorname{sym}\left\{\left[\begin{array}{lll}
\hat{e}_{1}^{H} & \widehat{e}_{10}^{H} & \widehat{e}_{17}^{H}
\end{array}\right] \widehat{V}\left(\widehat{e}_{1}-\widehat{e}_{17}\right)\right\} \\
& -\gamma^{2} \widehat{e}_{11}^{H} I \widehat{e}_{11}-\widehat{e}_{20}^{H} I \widehat{e}_{20}-\hat{e}_{14}^{H} \hat{\mathscr{G}} \widehat{e}_{14}+\varpi\left(\widehat{e}_{12}-\widehat{e}_{14}\right)^{H} \hat{\mathscr{G}}\left(\widehat{e}_{12}-\widehat{e}_{14}\right) \\
& \widehat{\Xi}_{1}=\operatorname{sym}\left\{\left(\widehat{e}_{1}-\widehat{e}_{17}\right)^{H} \widehat{Q}_{5} \widehat{e}_{10}\right\}+\widehat{e}_{10}\left[\operatorname{sym}\left(\widehat{\mathscr{V}}_{1}\right) \widehat{e}_{1}+\operatorname{sym}\left(\widehat{\mathscr{V}}_{2}-\widehat{\mathscr{V}}_{1}\right) \widehat{e}_{17}\right]+\Gamma_{8}^{H} \widehat{U} \Gamma_{8}, \\
& \xi_{4}^{H}(t)=\left[\dot{\vartheta}^{H}(t), \vartheta^{H}(t-\eta(t))\right], \\
& \xi_{5}^{H}(t)=\left[\vartheta^{H}(t), \vartheta^{H}(t-\sigma), \vartheta^{H}(t-\sigma(t)), \varphi^{H}(\vartheta(t)), \varphi^{H}(\vartheta(t-\sigma(t))), v_{1}^{H}, v_{2}^{H}, \dot{\vartheta}^{H}(t-\sigma(t)), \varphi^{H}(\vartheta(t-\sigma)), \dot{\vartheta}^{H}(t),\right. \\
& \left.\omega^{H}(t), \vartheta^{H}\left(t-\eta^{*}(t)\right), \vartheta^{H}(t-\eta), e^{H}\left(t-\eta^{*}(t)\right) v_{3}^{H}, v_{4}^{H}, \vartheta^{H}(t-\eta(t)), v_{5}^{H}, v_{6}^{H}\right] \\
& \xi_{6}^{H}(t)=\left[\vartheta(t)^{H}, \dot{\vartheta}^{H}(t), \vartheta(t-\eta(t))^{H}\right] \\
& \xi_{7}^{H}(t)=\left[\vartheta^{H}(t), \vartheta^{H}(t-\eta(t))\right] \\
& \widehat{\Lambda}=\left[-\widehat{\mathscr{X}} \mathscr{A}_{2 p}, 0,0, \widehat{\mathscr{X}} \mathscr{C}_{2 p}, \widehat{\mathscr{X}} \mathscr{D}_{2 p}, 0,0,0,0,0, \widehat{\mathscr{X}} \mathscr{H}_{1 p}, 0,0,0,0,0,0,0,0\right], \\
& \Gamma_{4}=\left[\begin{array}{c}
\widehat{e}_{1}-\widehat{e}_{17} \\
\widehat{e}_{1}+\widehat{e}_{17}-2 \widehat{e}_{15} \\
\widehat{e}_{17}-\widehat{e}_{13} \\
\widehat{e}_{17}+\widehat{e}_{13}-2 \widehat{e}_{16}
\end{array}\right] \\
& \Gamma_{5}=\left[\begin{array}{c}
\widehat{e}_{1}-\widehat{e}_{12} \\
\widehat{e}_{1}+\widehat{e}_{12}-2 \widehat{e}_{18} \\
\widehat{e}_{12}-\widehat{e}_{13} \\
\widehat{e}_{12}+\widehat{e}_{13}-2 \widehat{e}_{19}
\end{array}\right] \text {, } \\
& \Gamma_{6}=\left[\begin{array}{c}
\widehat{e}_{1} \\
\widehat{e}_{10} \\
\widehat{e}_{17}
\end{array}\right] \\
& \Gamma_{7}=\left[\begin{array}{c}
\hat{e}_{1} \\
\widehat{e}_{17}
\end{array}\right] \text {, } \\
& \Gamma_{8}=\left[\begin{array}{l}
\widehat{e}_{10} \\
\widehat{e}_{17}
\end{array}\right], \\
& \widehat{e}_{p}=\left[\begin{array}{lll}
0_{n \times(p-1) n} & I_{n} & 0_{n \times(19-p) n}
\end{array}\right] .
\end{aligned}
$$


System (8) possessing $H \infty$ performance is asymptotically stable under the SBET scheme (6), and the controller gain matrices in (7) are designed as $\widetilde{\mathscr{K}}_{p}=Y_{2 p} \widehat{\mathscr{X}}^{-1}$.
Proof. Choose the following LKF:

$$
\begin{aligned}
V(\vartheta(t), t, p)= & \sum_{m=1}^{5} V_{m}(\vartheta(t), t, p), \\
V_{1}(\mathcal{\vartheta}(t), t, p)= & \vartheta^{H}(t) \mathscr{P}_{p} \mathcal{\vartheta}(t)+\int_{t-\sigma}^{t} \xi_{2}^{H}(s) \mathscr{R}_{p} \xi_{2}(s) \mathrm{d} s+\int_{t-\sigma(t)}^{t} \dot{\vartheta}^{H}(s) \mathscr{Q}_{1} \dot{\vartheta}(s) \mathrm{d} s+\int_{t-\sigma(t)}^{t} \vartheta^{H}(s) \mathscr{Q}_{2} \mathcal{\vartheta}(s) \mathrm{d} s \\
& +\sigma \int_{\sigma}^{0} \int_{t-\theta}^{t} \dot{\vartheta}^{H}(s) \mathscr{Q}_{3} \dot{\vartheta}(s) \mathrm{d} s \mathrm{~d} \theta+\int_{t-\sigma}^{t} \int_{\theta}^{0} \xi_{2}^{H}(s) \mathscr{R} \xi_{2}(s) \mathrm{d} s \mathrm{~d} \theta \\
V_{2}(\vartheta(t), t, p)= & \eta \int_{\eta}^{0} \int_{t-\theta}^{t} \dot{\vartheta}^{H}(s) \mathscr{Q}_{4} \dot{\vartheta}(s) \mathrm{d} s \mathrm{~d} \theta \\
V_{3}(\mathcal{\vartheta}(t), t, p)= & \eta \int_{\eta}^{0} \int_{t-\theta}^{t} \dot{\vartheta}^{H}(s) \mathbb{Q}_{6} \dot{\vartheta}(s) \mathrm{d} s \mathrm{~d} \theta, \\
V_{4}(\vartheta(t), t, p)= & \left(h_{k}-\eta(t)\right) \int_{t-\eta(t)}^{t} \xi_{4}^{H}(s) U \xi_{4}(s) \mathrm{d} s, \\
V_{5}(\vartheta(t), t, p)= & \left(h_{k}-\eta(t)\right)[\vartheta(t)-\vartheta(t-\eta(t))]^{H} \mathscr{Q}_{5}[\vartheta(t)-\vartheta(t-\eta(t))] \\
& +\left(h_{k}-\eta(t)\right) \xi_{7}^{H}(t) \mathscr{V} \xi_{7}(t),
\end{aligned}
$$

with

$$
\begin{aligned}
& h_{k}=h+\varsigma\left(\left(\iota_{k}+j+1\right) h\right)-\varsigma\left(\left(\iota_{k}+j\right) h\right) \text {, } \\
& \mathscr{P}_{p}=\left(\widehat{\mathscr{X}}^{H}\right)^{-1} \widehat{\mathscr{P}}_{p} \widehat{\mathscr{X}}^{-1} \text {, } \\
& \widehat{Q}_{p}=\left(\hat{X}^{H}\right)^{-1} \widehat{Q}_{p} \hat{X}^{-1} \text {, } \\
& \widehat{Q}_{4}=\left(\widehat{X}^{H}\right)^{-1} \widehat{Q}_{4} \widehat{x}^{-1} \text {, } \\
& \widehat{Q}_{5}=\left(\widehat{X}^{H}\right)^{-1} \widehat{Q}_{5} \widehat{x}^{-1} \text {, } \\
& \mathscr{R}_{p}=\left(\widehat{\mathscr{X}}^{H}\right)^{-1} \widehat{\mathscr{R}}_{p} \widehat{\mathscr{X}}^{-1} \text {, } \\
& \mathscr{R}=\left(\widehat{\mathscr{X}}^{H}\right)^{-1} \widehat{\mathscr{R}} \widehat{\mathscr{X}}^{-1}, \\
& p \in\{1,2,3\} \text {, }
\end{aligned}
$$

being positive defined Hermitian matrices with proper dimension, and

$$
\begin{aligned}
& U=\left[\begin{array}{cc}
U_{11} & U_{12} \\
* & U_{22}
\end{array}\right], \\
& \mathscr{V}=\left[\begin{array}{cc}
\operatorname{sym}\left(\frac{\mathscr{V}_{1}}{2}\right) & -\mathscr{V}_{1}+\mathscr{V}_{2} \\
* & \operatorname{sym}\left(\frac{\mathscr{V}_{1}}{2}-\mathscr{V}_{2}\right)
\end{array}\right] .
\end{aligned}
$$

From the definition of $V(\mathcal{\vartheta}(t), p)$, noting $V_{\mathcal{F}}(\vartheta(t), p), \mathcal{J}=1,2,3$, is a continuous function; it is easy to see that

$$
\lim _{t \longrightarrow\left(\left(\iota_{k}+j\right) h+\varsigma\left(\left(\iota_{k}+j\right) h\right)\right)^{-}} V_{\mathcal{F}}(\mathcal{Y}(t), p)=V_{\mathcal{F}}\left(\mathcal{V}\left(\left(\iota_{k}+j\right) h+\varsigma\left(\left(\iota_{k}+j\right) h\right)\right), p\right) .
$$

Also, we can derive that

$$
\begin{aligned}
& \lim _{t \longrightarrow\left(\left(\iota_{k}+j\right) h+\zeta\left(\left(\iota_{k}+j\right) h\right)\right)^{-}} V_{4}(\vartheta(t), p)= \\
& t \longrightarrow\left(\left(\iota_{\left.k+j) h+\zeta\left(\left(\iota_{k}+j\right) h\right)\right)^{+}} V_{4}(\vartheta(t), p)=V_{4}\left(\vartheta\left(\left(\iota_{k}+j\right) h+\varsigma\left(\left(\iota_{k}+j\right) h\right)\right), p\right)=0,\right.\right. \\
& \lim _{t \longrightarrow\left(\left(\iota_{k}+j\right) h+\varsigma\left(\left(\iota_{k}+j\right) h\right)\right)^{-}} V_{5}(\vartheta(t), p)=\lim _{t \longrightarrow\left(\left(\iota_{k}+j\right) h+\zeta\left(\left(\iota_{k}+j\right) h\right)\right)^{+}} V_{5}(\vartheta(t), p)=V_{5}\left(\vartheta\left(\left(\iota_{k}+j\right) h+\varsigma\left(\left(\iota_{k}+j\right) h\right)\right), p\right)=0 .
\end{aligned}
$$


Therefore, $V(\vartheta(t), p)$ is continuous at $t=\left(l_{k}+j\right) h+$ $\varsigma\left(\left(\iota_{k}+j\right) h\right)$. Moreover, $V_{\mathcal{f}}(\vartheta(t), p) \geq 0$, that $V(\vartheta(t), p)=$ $V_{\mathcal{g}}(\vartheta(t), p)+V_{4}(\vartheta(t), p)+V_{5}(\vartheta(t), p) \geq 0$ for any $k$. By pre- and postmultiplying $\left(\widehat{X}^{H}\right)^{-1}$ and $\widehat{x}^{-1}$ with (60), we have

$$
\begin{aligned}
& \sum_{q \in \aleph_{k}^{p}}^{n} \pi_{p q}\left(\mathscr{R}_{q}-\mathscr{M}_{p}\right) \leq \mathscr{R}, \\
& \mathscr{P}_{q}-\mathscr{W}_{p} \leq 0, q \in \aleph_{u k}^{p}, p \neq q, \mathscr{R}_{q}-\mathscr{M}_{p} \leq 0, q \in \aleph_{u k}^{p}, p \neq q, \\
& \mathscr{P}_{q}-\mathscr{W}_{p} \geq 0, q \in \aleph_{u k}^{p}, p=q, \mathscr{R}_{q}-\mathscr{M}_{p} \geq 0, q \in \aleph_{u k}^{p}, p=q,
\end{aligned}
$$

and following the same procedure to Theorem 1, we have

$$
\begin{aligned}
L V_{1}(\vartheta(t), p) \leq & \vartheta^{H}(t) \mathscr{P}_{p} \dot{\vartheta}(t)+\dot{\vartheta}^{H}(t) \mathscr{P}_{p} \vartheta(t)+\vartheta^{H}(t) \sum_{q \in \mathbb{N}_{k}^{p}} \pi_{p q}\left(\mathscr{P}_{q}-\mathscr{W}_{p}\right) \vartheta(t)+\xi_{2}^{H}(t) \mathscr{R}_{p} \xi_{2}(t) \\
& -\xi_{2}^{H}(t-\sigma) \mathscr{R}_{p} \xi_{2}(t-\sigma)+\dot{\vartheta}^{H}(t)\left(\mathscr{Q}_{1}+\sigma^{2} \mathscr{Q}_{3}\right) \dot{\vartheta}(t)-\left(1-\sigma_{d}\right) \dot{\vartheta}^{H}(t-\sigma(t)) \mathscr{Q}_{1} \dot{\vartheta}(t-\sigma(t))+\vartheta^{H}(t) \mathscr{Q}_{2} \vartheta(t) \\
& -\left(1-\sigma_{d}\right) \vartheta^{H}(t-\sigma(t)) \mathscr{Q}_{2} \vartheta(t-\sigma(t))-\xi_{5}^{H}(t) \Gamma_{1}^{H} \bar{Q}_{1} \Gamma_{1} \xi_{5}(t)+\sigma \xi_{2}^{H}(t) \mathscr{R}_{2}(t)+\vartheta^{H}(t) L E_{1} L \vartheta(t) \\
& -\varphi^{H}(\vartheta(t)) E_{1} \varphi(\vartheta(t))+\vartheta^{H}(t-\sigma(t)) L E_{2} L \vartheta(t-\sigma(t))-\varphi^{H}(\vartheta(t-\sigma(t))) E_{2} \varphi(\vartheta(t-\sigma(t))) .
\end{aligned}
$$

The following inequalities can be obtained because of Lemma 3 with arbitrary matrices $\mathcal{S}_{33}, \mathcal{S}_{44}, \mathcal{S}_{34}, \mathcal{S}_{55}, \mathcal{S}_{66}$, $\mathcal{S}_{56} \in \mathscr{C}^{n \times n}:$

$$
\begin{aligned}
& L V_{2}(\mathcal{\vartheta}(t), p)=\eta^{2} \dot{\vartheta}^{H}(t) \mathbb{Q}_{4} \dot{\vartheta}(t)-\eta \int_{t-\eta}^{t} \dot{\vartheta}^{H}(s) \mathscr{Q}_{4} \dot{\vartheta}(s) \mathrm{d} s=\eta^{2} \dot{\vartheta}^{H}(t) \mathbb{Q}_{4} \dot{\vartheta}(t)-\eta \int_{t-\eta(t)}^{t} \dot{\vartheta}^{H}(s) Q_{4} \dot{\vartheta}(s) \mathrm{d} s \\
& -\eta \int_{t-\eta}^{t-\eta(t)} \dot{\vartheta}^{H}(s) Q_{4} \dot{\vartheta}(s) \mathrm{d} s \leq \eta^{2} \dot{\vartheta}^{H}(t) \mathscr{Q}_{4} \dot{\vartheta}(t)-\xi_{5}^{H}(t) \Gamma_{4}^{H} \mathscr{Q}_{2} \Gamma_{4} \xi_{5}(t) \\
& L V_{3}(\vartheta(t), p)=\eta^{2} \dot{\vartheta}^{H}(t) Q_{6} \dot{\vartheta}(t)-\eta \int_{t-\eta}^{t} \dot{\vartheta}^{H}(s) Q_{6} \dot{\vartheta}(s) \mathrm{d} s=\eta^{2} \dot{\vartheta}^{H}(t) \mathbb{Q}_{6} \dot{\vartheta}(t)-\eta \int_{t-\eta^{*}(t)}^{t} \dot{\vartheta}^{H}(s) Q_{6} \dot{\vartheta}(s) \mathrm{d} s \\
& -\eta \int_{t-\eta}^{t-\eta^{*}(t)} \dot{\vartheta}^{H}(s) Q_{6} \dot{\vartheta}(s) \mathrm{d} s \leq \eta^{2} \dot{\vartheta}^{H}(t) Q_{6} \dot{\vartheta}(t)-\xi_{5}^{H}(t) \Gamma_{5}^{H} Q_{3} \Gamma_{5} \xi_{5}(t), \\
& L V_{4}(\vartheta(t), p)=\left(h_{k}-\eta(t)\right) \xi_{4}^{H} U \xi_{4}-\int_{t-\eta(t)}^{t} \xi_{4}^{H} U \xi_{4} \mathrm{~d} s=\left(h_{k}-\eta(t)\right) \xi_{4}^{H} U \xi_{4}-\int_{t-\eta(t)}^{t} \dot{\vartheta}^{H}(s) U_{11} \dot{\vartheta}(s) \mathrm{d} s \\
& -\int_{t-\eta(t)}^{t} \dot{\vartheta}^{H}(s) \mathrm{d} s U_{12} \vartheta(t-\eta(t))-\vartheta^{H}(t-\eta(t)) U_{12} \int_{t-\eta(t)}^{t} \dot{\vartheta}(s) \mathrm{d} s-\eta(t) \vartheta^{H}(t-\eta(t)) U_{22} \vartheta(t-\eta(t)) .
\end{aligned}
$$

From the corollary of Lemma 4, the following formula is established with $V$ of appropriate dimensions:

$$
-\int_{t-\eta(t)}^{t} \dot{\vartheta}^{H}(s) U_{11} \dot{\vartheta}(s) \mathrm{d} s \leq \eta(t) \xi_{6}^{H}(t) V^{H} U_{11}^{-1} V \xi_{6}(t)+\xi_{6}^{H}(t) V^{H} \int_{t-\eta(t)}^{t} \dot{\vartheta}(s) \mathrm{d} s+\int_{t-\eta(t)}^{t} \dot{\vartheta}(s) \mathrm{d} s V \xi_{6}(t) .
$$

Then, we have 


$$
\begin{aligned}
L V_{4}(\vartheta(t), p)= & \left(h_{k}-\eta(t)\right) \xi_{4}^{H} U \xi_{4}-\int_{t-\eta(t)}^{t} \xi_{4}^{H} U \xi_{4} \mathrm{~d} s \leq\left(h_{k}-\eta(t)\right) \xi_{4}^{H} U \xi_{4}+\eta(t) \xi_{6}^{H}(t) V^{H} U_{11}^{-1} V \xi_{6}(t) \\
& +\xi_{6}^{H}(t) V^{H} \int_{t-\eta(t)}^{t} \dot{\vartheta}(s) \mathrm{d} s+\int_{t-\eta(t)}^{t} \dot{\vartheta}(s) \mathrm{d} s V \xi_{6}(t)-\eta(t) \vartheta^{H}(t-\eta(t)) U_{22} \vartheta(t-\eta(t)) \\
& -\int_{t-\eta(t)}^{t} \dot{\vartheta}^{H}(s) \mathrm{d} s U_{12} \vartheta(t-\eta(t))-\vartheta^{H}(t-\eta(t)) U_{12} \int_{t-\eta(t)}^{t} \dot{\vartheta}(s) \mathrm{d} s .
\end{aligned}
$$

Noticing that $\eta(t)=t-\left(\iota_{k}+j\right) h-\varsigma\left(\left(\iota_{k}+j\right) h\right)$, the following inequality is obtained:

$$
\begin{aligned}
L V_{5}(\vartheta(t), p) \leq & -[\vartheta(t)-\vartheta(t-\eta(t))]^{H} \mathscr{Q}_{5}[\vartheta(t)-\vartheta(t-\eta(t))]-\xi_{7}^{H}(s) \mathscr{V}_{7}(s) \\
& +\left(h_{k}-\eta(t)\right)[\vartheta(t)-\vartheta(t-\eta(t))]^{H} \mathscr{Q}_{5} \dot{\vartheta}(t)+\left(h_{k}-\eta(t)\right) \dot{\vartheta}^{H}(t) \mathscr{Q}_{5}[\vartheta(t)-\vartheta(t-\eta(t))] \\
& +\left(h_{k}-\eta(t)\right)\left[\dot{\vartheta}^{H}(t)\left(\mathscr{V}_{1}+\mathscr{V}_{1}^{H}\right) \vartheta(t)+\left(h_{k}-\eta(t)\right) \dot{\vartheta}^{H}(t)\left(\mathscr{V}_{2}-\mathscr{V}_{1}\right) \vartheta(t-\eta(t))\right] \\
& +\left(h_{k}-\eta(t)\right) \vartheta^{H}(t-\eta(t))\left(\mathscr{V}_{2}-\mathscr{V}_{1}\right) \dot{\vartheta}(t) .
\end{aligned}
$$

From the event-triggered mechanism (6), when $t \in \mathscr{T}_{k}$, it is clear that

$$
e^{H}\left(\iota_{k} h+l h\right) \mathscr{G} e\left(\iota_{k} h+l h\right)<\omega\left[\vartheta\left(\iota_{k} h+l h\right)-e\left(\iota_{k} h+l h\right)\right]^{H} \mathscr{G}\left[\vartheta\left(\iota_{k} h+l h\right)-e\left(\iota_{k} h+l h\right)\right] .
$$

It means that the data of the current sampling moment is not transmitted. Moreover, one can obtain the following zero equality based on system (8) with any nonsingular matrix $\mathscr{X}_{4}$ with appropriate dimension, defining $\mathscr{X}_{3}=\rho \mathscr{X}_{4}$, that $\mathscr{X}_{4}$ is nonsingular:

$$
\begin{aligned}
0= & \left(\vartheta^{H}(t) \mathscr{X}_{3}^{H}+\dot{\vartheta}^{H}(t) \mathscr{X}_{4}^{H}\right)\left(-\dot{\vartheta}(t)-\mathscr{A}_{1 p} \vartheta(t)+\mathscr{B}_{1 p} \dot{\vartheta}(t-\sigma(t))+\mathscr{C}_{1 p} \varphi(\vartheta(t))+\mathscr{D}_{1 p} \varphi(\vartheta(t-\sigma(t)))+\mathrm{E}_{1 p} \omega(t)\right. \\
& \left.+\mathscr{E}_{2 p} \tilde{\mathscr{K}}_{p} \vartheta(t-\eta *(t))-\mathscr{E}_{2 p} \tilde{\mathscr{K}}_{p} e(t-\eta *(t))\right)+\left(-\dot{\vartheta}(t)-\mathscr{A}_{1 p} \vartheta(t)+\mathscr{B}_{1 p} \dot{\vartheta}(t-\sigma(t))+\mathscr{C}_{1 p} \varphi(\vartheta(t))\right. \\
& \left.+\mathscr{D}_{1 p} \varphi(\vartheta(t-\sigma(t)))+\mathscr{E}_{1 p} \omega(t)+\mathscr{E}_{2 p} \tilde{\mathscr{K}}_{p} \vartheta\left(t-\eta^{*}(t)\right)-\mathscr{E}_{2 p} \tilde{\mathscr{K}}_{p} e\left(t-\eta^{*}(t)\right)\right)^{H}\left(\mathscr{X}_{3} \vartheta(t)+\mathscr{X}_{4} \dot{\vartheta}(t)\right) .
\end{aligned}
$$

Combining with (69)-(76), we have the following equation:

$$
\begin{aligned}
L V & (\vartheta(t), p)+v^{H}(t) v(t)-\gamma^{2} \omega^{H}(t) \omega(t) \leq L V(\vartheta(t), p)+v^{H}(t) v(t)-\gamma^{2} \omega^{H}(t) \omega(t)-e^{H}\left(t_{k} h+l h\right) \mathscr{G}_{e}\left(t_{k} h+l h\right) \\
& +\Phi\left[\vartheta\left(\iota_{k} h+l h\right)-e\left(\iota_{k} h+l h\right)\right]^{H} \mathscr{G}\left[\vartheta\left(\iota_{k} h+l h\right)-e\left(\iota_{k} h+l h\right)\right]+\operatorname{sym}\left\{( \vartheta ^ { H } ( t ) \mathscr { X } _ { 3 } ^ { H } + \dot { \vartheta } ^ { H } ( t ) \mathscr { X } _ { 4 } ^ { H } ) \left(-\dot{\vartheta}(t)-\mathscr{A}_{1 p} \vartheta(t)\right.\right. \\
& \left.\left.+\mathscr{B}_{1 p} \dot{\vartheta}(t-\sigma(t))+\mathscr{C}_{1 p} \varphi(\vartheta(t))+\mathscr{D}_{1 p} \varphi(\vartheta(t-\sigma(t)))+\mathscr{E}_{1 p} \omega(t)+\mathscr{E}_{2 p} \tilde{\mathscr{K}}_{p} \vartheta(t-\eta(t))-\mathscr{E}_{2 p} \tilde{\mathscr{K}}_{p} e(t-\eta(t))\right)\right\} .
\end{aligned}
$$

By dividing the right-hand side of the above inequality into three parts, we have 


$$
\begin{aligned}
& L V(\vartheta(t), p)+v^{H}(t) v(t)-\gamma^{2} \omega^{H}(t) \omega(t) \leq \xi_{5}^{H}(t)\left(\widetilde{\Xi}+\left(h_{k}-\eta(t)\right) \widetilde{\Xi}_{1}+\eta(t) \widetilde{\Xi}_{2}+\varrho^{H} \varrho\right) \xi_{5}(t) \\
& =\xi_{5}^{H}(t)\left[\frac{h_{k}-\eta(t)}{h_{k}} \Omega_{2}\left(h_{k}\right)+\frac{\eta(t)}{h_{k}} \Omega_{3}\left(h_{k}\right)\right] \xi_{5}(t),
\end{aligned}
$$

with

$$
\begin{aligned}
& \Omega_{2}\left(h_{k}\right)=\widetilde{\Xi}+h_{k} \widetilde{\Xi}_{1}+\varrho^{H} \varrho \text {, } \\
& \Omega_{3}\left(h_{k}\right)=\widetilde{\Xi}+h_{k} \widetilde{\Xi}_{2}+\varrho^{H} \varrho \text {, } \\
& \widetilde{\Xi}=\operatorname{sym}\left\{\left(\widehat{e}_{1}^{H} \mathscr{X}_{3}^{H}+\widehat{e}_{10}^{H} \mathscr{X}_{4}^{H}\right)\left(-\widehat{e}_{10}-\mathscr{A}_{1 p} \widehat{e}_{1}+\mathscr{B}_{1 p} \widehat{e}_{8}+\mathscr{C}_{1 p} \widehat{e}_{4}+\mathscr{D}_{1 p} \widehat{e}_{5}+\mathscr{E}_{1 p} \widehat{e}_{9}+\mathscr{E}_{2 p} \widetilde{\mathscr{K}}_{p}\left(\widehat{e}_{12}-\widehat{e}_{14}\right)\right)\right\} \\
& +\operatorname{sym}\left\{\widehat{e}_{1}^{H} \mathscr{P}_{p} \widehat{e}_{10}\right\}+\widehat{e}_{1}^{H} \sum_{q \in \aleph_{k}^{p}}^{n} \pi_{p q}\left(\mathscr{P}_{q}-\mathscr{W}_{p}\right) \widehat{e}_{1}+\Gamma_{2}^{H} \mathscr{R}_{p} \Gamma_{2}+\Gamma_{3}^{H} \mathscr{R}_{p} \Gamma_{3}+\widehat{e}_{10}^{H} \widehat{Q}_{1} \widehat{e}_{10}-\left(1-\sigma_{d}\right) \widehat{e}_{8}^{H} Q_{1} \widehat{e}_{8} \\
& +\widehat{e}_{1}^{H} Q_{2} \widehat{e}_{1}-\left(1-\sigma_{d}\right) \widehat{e}_{3}^{H} Q_{2} \widehat{e}_{3}+\sigma^{2} \widehat{e}_{10}^{H} Q_{3} \widehat{e}_{10}-\Gamma_{1}^{H} Q_{1} \Gamma_{1}+\sigma \Gamma_{2}^{H} \mathscr{R} \Gamma_{2}+\widehat{e}_{1}^{H} L^{H} E_{1} L \widehat{e}_{1}-\widehat{e}_{4}^{H} E_{1} \widehat{e}_{4} \\
& +\widehat{e}_{3}^{H} L^{H} E_{2} L \widehat{e}_{3}-\widehat{e}_{5}^{H} E_{2} \widehat{e}_{5}+\eta^{2} \widehat{e}_{10}^{H}\left(\widehat{Q}_{4}+\widehat{Q}_{6}\right) \widehat{e}_{10}-\Gamma_{4}^{H} Q_{2} \Gamma_{4}-\Gamma_{5}^{H} Q_{3} \Gamma_{5}-\left(\widehat{e}_{1}-\widehat{e}_{17}\right)^{H} Q_{5}\left(\widehat{e}_{1}-\widehat{e}_{17}\right) \\
& -\Gamma_{7}^{H} \mathscr{V} \Gamma_{7}-\operatorname{sym}\left\{\left(\widehat{e}_{1}-\widehat{e}_{17}\right)^{H} U_{12} \widehat{e}_{17}\right\}+\operatorname{sym}\left\{\left[\begin{array}{lll}
\hat{e}_{1}^{H} & \widehat{e}_{10}^{H} & \widehat{e}_{17}^{H}
\end{array}\right] V\left(\widehat{e}_{1}-\widehat{e}_{17}\right)\right\}-\gamma^{2} \widehat{e}_{11}^{H} I \widehat{e}_{11} \\
& -\widehat{e}_{14}^{H} \mathscr{G} \widehat{e}_{14}+\omega\left(\widehat{e}_{12}-\widehat{e}_{14}\right)^{H} \mathscr{G}\left(\widehat{e}_{12}-\widehat{e}_{14}\right) \text {, } \\
& \widetilde{\Xi}_{1}=\operatorname{sym}\left\{\left(\widehat{e}_{1}-\widehat{e}_{17}\right)^{H} \mathscr{Q}_{5} \widehat{e}_{10}\right\}+\widehat{e}_{10}\left[\operatorname{sym}\left(\mathscr{V}_{1}\right) \widehat{e}_{1}+\operatorname{sym}\left(\mathscr{V}_{2}-\mathscr{V}_{1}\right) \widehat{e}_{17}\right]+\Gamma_{8}^{H} U \Gamma_{8} \text {, } \\
& \widetilde{\Xi}_{2}=-\widehat{e}_{17}^{H} U_{22} \widehat{e}_{17}+\Gamma_{6}^{H} V U_{11}^{-1} V^{H} \Gamma_{6} \text {, } \\
& \varrho=-\mathscr{A}_{2 p} \widehat{e}_{1}+\mathscr{C}_{2 p} \widehat{e}_{4}+\mathscr{D}_{2 p} \widehat{e}_{5}+\mathscr{H}_{1 p} \widehat{e}_{11} \text {. }
\end{aligned}
$$

Define

$$
\begin{aligned}
& \widehat{\mathscr{X}}=\mathscr{X}_{4}^{-1}, Y_{2 p}=\widetilde{\mathscr{X}}_{p} \widehat{\mathscr{X}}, \widetilde{V}=\widehat{\mathscr{X}}^{H} V^{H}, \widehat{\mathfrak{X}}_{\hbar}=\operatorname{diag}\{\underbrace{\widehat{\mathscr{X}}, \widehat{\mathscr{X}}, \ldots, \widehat{\mathscr{X}}}_{\hbar}\}, \widehat{\mathscr{P}}_{p}=\widehat{\mathscr{X}}^{H} \mathscr{P}_{p}, \widehat{\mathscr{X}}, \widehat{\mathscr{W}}_{p}=\widehat{\mathscr{X}}^{H} \mathscr{W}_{p}, \widehat{\mathscr{X}}, \\
& \widehat{\mathscr{M}}_{p}=\widehat{\mathscr{X}}^{H} \mathscr{M}_{p} \widehat{\mathscr{X}}, \widehat{\mathscr{P}}_{q}=\widehat{\mathscr{X}}^{H} \mathscr{P}, \widehat{\mathscr{X}}, \widehat{\mathscr{R}}_{q}=\widehat{\mathscr{X}}^{H} \mathscr{R} q \widehat{\mathscr{X}}, \widehat{\mathscr{R}}_{p}=\widehat{\mathscr{X}}^{H} \mathscr{R}_{p} \widehat{\mathscr{X}}, \widehat{Q}_{p}=\widehat{\mathscr{X}}^{H} \widehat{Q}_{p} \widehat{\mathscr{X}}, \widehat{Q}_{4}=\widehat{\mathscr{X}}^{H} \widehat{Q}_{4} \widehat{\mathscr{X}}, \\
& \widehat{Q}_{5}=\widehat{\mathscr{X}}^{H} Q_{5} \widehat{\mathscr{X}}, \widehat{Q}_{6}=\widehat{\mathscr{X}}^{H} Q_{6} \widehat{\mathscr{X}}, \widehat{\mathscr{R}}_{p}=\widehat{\mathscr{X}}^{H} \mathscr{R}_{p} \widehat{\mathscr{X}}, \widehat{\mathscr{E}}_{1}=\widehat{\mathscr{X}}^{H} \mathscr{E}_{1} \widehat{\mathscr{X}}, \hat{\mathscr{E}}_{2}=\widehat{\mathscr{X}}^{H} \mathscr{E}_{2} \widehat{\mathscr{X}}, \hat{\mathcal{S}}_{11}=\widehat{\mathscr{X}}^{H} \mathcal{S}_{11} \widehat{\mathscr{X}}, \hat{\mathcal{S}}_{12}=\widehat{\mathscr{X}}^{H} \mathcal{S}_{12} \widehat{\mathscr{X}}, \\
& \hat{\mathcal{S}}_{22}=\widehat{\mathscr{X}}^{H} \mathcal{S}_{22} \widehat{\mathscr{X}}, \hat{\mathcal{S}}_{33}=\widehat{\mathscr{X}}^{H} \mathcal{S}_{33} \widehat{\mathscr{X}}, \hat{\mathcal{S}}_{34}=\widehat{\mathscr{X}}^{H} \mathcal{S}_{34} \widehat{\mathscr{X}}, \hat{\mathcal{S}}_{44}=\widehat{\mathscr{X}}^{H} \mathcal{S}_{44} \widehat{\mathscr{X}}, \hat{\mathcal{S}}_{55}=\widehat{\mathscr{X}}^{H} \mathcal{S}_{55} \widehat{\mathscr{X}} \text {, } \\
& \widehat{\mathcal{S}}_{56}=\widehat{\mathscr{X}}^{H} \mathcal{S}_{56} \widehat{\mathscr{X}}, \hat{\mathcal{S}}_{66}=\widehat{\mathscr{X}}^{H} \mathcal{S}_{66} \widehat{\mathscr{X}}, \widehat{U}_{11}=\widehat{\mathscr{X}}^{H} U_{11} \widehat{\mathscr{X}}, \widehat{U}_{12}=\widehat{\mathscr{X}}^{H} U_{12} \widehat{\mathscr{X}}, \widehat{U}_{22}=\widehat{\mathscr{X}}^{H} U_{22} \widehat{\mathscr{X}}, \widehat{I}=\widehat{x}^{H} I \widehat{x} \text {, } \\
& \widehat{\mathscr{G}}=\widehat{\mathscr{X}}^{H} \hat{\mathrm{GX}}, \hat{\mathscr{V}}=\widehat{\mathscr{X}}^{H} \hat{\mathscr{X}}, \hat{\mathscr{V}}_{1}=\widehat{\mathscr{X}}^{H} \mathscr{V}_{1} \widehat{\mathscr{X}}, \widehat{\mathscr{V}}_{2}=\widehat{\mathscr{X}}^{H} \mathscr{V}_{2} \widehat{\mathscr{X}}, \widehat{U}=\widehat{\mathscr{X}}^{H} U \widehat{\mathscr{X}}, \widehat{V}=\widehat{\mathscr{X}}^{H} V \widehat{\mathscr{X}}
\end{aligned}
$$

and then, by pre- and postmultiplying $\widehat{\mathfrak{X}}_{\hbar}^{H}$ and $\widehat{\mathfrak{X}}_{\hbar}(\hbar=19)$ with $\Omega_{2}\left(h_{k}\right)$ and $\Omega_{3}\left(h_{k}\right)$, combining (57)-(61) and (78), then applying Schur complement, we have $\widetilde{\Xi}+h_{k} \widetilde{\Xi}_{1}+\varrho^{H} \varrho<0, \widetilde{\Xi}+h_{k} \widetilde{\Xi}_{2}+\varrho^{H} \varrho<0$, that $L V(\vartheta(t), p)+$ $v^{H}(t) v(t)-\gamma^{2} \omega^{H}(t) \omega(t)<0$ for $t \in \mathscr{T}_{k}$. Therefore, if the LMIs in Theorem 2 hold, according to the similar proof line as Theorem 1, the CVNNs (8) with Markovian jump are asymptotically stable and possess $H \infty$ performance based on a SBET controller; this completes the proof.
Remark 4. As far as we know, a discrete sampling-based event-triggered controller which is designed for the neutral-type CVNNs with partly unknown Markov jump in this work has not received much attention, which reduces the data transmission rate greatly for it only needs to monitor the state of our system at the discrete moment. Also, the partial unknown Markov phenomenon is taken into account which makes the results more general and practical. 
Remark 5. The segmented sawtooth structure caused by sampling hold is fully considered by $V_{4}(\vartheta(t), p)$ and $V_{5}(\vartheta(t), p)$ that the conservatism of our results would be reduced. Furthermore, we analyze the CVNNs under the sampling-based event-driven framework while not dividing it into the real part and the imaginary part; it is able to reduce computational complexity greatly while the desired effect is achieved.
Remark 6. $V_{2}(\vartheta(t), p)$ and $V_{3}(\vartheta(t), p)$ in Theorem 2 have a very similar presentation, but in fact, they are different, and different interval segmentations are adopted for the processing of the two LKFs, which can also be merged together as $V_{6}(\vartheta(t), t, p)=\eta \int_{\eta}^{0} \int_{t-\theta}^{t} \dot{\vartheta}^{H}(s) Q_{7} \dot{\vartheta}(s) \mathrm{d} s \mathrm{~d} \theta$ :

$$
\begin{aligned}
L V_{6}(\vartheta(t), t, p)= & \eta^{2} \dot{\vartheta}^{H}(t) Q_{7} \dot{\vartheta}(t)-\eta \int_{t-\eta}^{t} \dot{\vartheta}^{H}(s) Q_{7} \dot{\vartheta}(s) \mathrm{d} s \\
= & \eta^{2} \dot{\vartheta}^{H}(t) Q_{7} \dot{\vartheta}(t)-\eta \int_{t-\eta(t)}^{t} \dot{\vartheta}^{H}(s) Q_{7} \dot{\vartheta}(s) \mathrm{d} s \\
& -\eta \int_{t-\eta^{*}(t)}^{t-\eta(t)} \dot{\vartheta}^{H}(s) Q_{7} \dot{\vartheta}(s) \mathrm{d} s-\eta \int_{t-\eta}^{t-\eta^{*}(t)} \dot{\vartheta}^{H}(s) Q_{7} \dot{\vartheta}(s) \mathrm{d} s .
\end{aligned}
$$

According to Lemma 1,

$$
\begin{aligned}
& L V_{6}(\vartheta(t), t, p) \leq \eta^{2} \dot{\vartheta}^{H}(t) Q_{7} \dot{\vartheta}(t)-\frac{\eta}{\eta(t)} \alpha_{1}^{H} Q_{7} \alpha_{1} \\
&-\frac{\eta}{\eta^{*}(t)-\eta(t)} \alpha_{2}^{H} Q_{7} \alpha_{2}-\frac{\eta}{\eta-\eta^{*}(t)} \alpha_{3}^{H} Q_{7} \alpha_{3} \\
& \leq \eta^{2} \dot{\vartheta}^{H}(t) Q_{7} \dot{\vartheta}(t)-\left[\begin{array}{c}
\alpha_{1} \\
\alpha_{2} \\
\alpha_{3}
\end{array}\right]\left[\begin{array}{ccc}
Q_{7} & S_{1} & S_{2} \\
* & Q_{7} & S_{3} \\
* & * & Q_{7}
\end{array}\right]\left[\begin{array}{l}
\alpha_{1} \\
\alpha_{2} \\
\alpha_{3}
\end{array}\right],
\end{aligned}
$$

with

$$
\begin{aligned}
& \alpha_{1}=\vartheta(t)-\vartheta(t-\eta(t)), \\
& \alpha_{2}=\vartheta(t-\eta(t))-\vartheta\left(t-\eta^{*}(t)\right), \\
& \alpha_{3}=\vartheta\left(t-\eta^{*}(t)\right)-\vartheta(t),
\end{aligned}
$$

$S_{1}, S_{2}, S_{3} \in \mathscr{C}^{n \times n}$.
However, the vectors that are contained in $L V_{6}(\vartheta(t), t, p)$ are not as comprehensive as those in $L V_{2}(\vartheta(t), t, p)$ and $L V_{3}(\vartheta(t), t, p)$.

\section{Numerical Examples}

Through numerical simulations, two examples are given to demonstrate the superiority and effectiveness of our conclusions derived from previous chapters. 
Example 1. Consider the CVNNs (5) with the following parameters:

$$
\begin{aligned}
& \mathscr{A}_{11}=\left(\begin{array}{cc}
0.8 & 0 \\
0 & 0.7
\end{array}\right) \text {, } \\
& \mathscr{A}_{12}=\left(\begin{array}{cc}
0.6 & 0 \\
0 & 0.5
\end{array}\right) \text {, } \\
& \mathscr{A}_{13}=\left(\begin{array}{cc}
0.5 & 0 \\
0 & 0.4
\end{array}\right) \text {, } \\
& \mathscr{A}_{21}=\left(\begin{array}{cc}
0.6 & 0 \\
0 & 0.5
\end{array}\right) \text {, } \\
& \mathscr{A}_{22}=\left(\begin{array}{cc}
0.7 & 0 \\
0 & 0.6
\end{array}\right) \text {, } \\
& \mathscr{A}_{23}=\left(\begin{array}{cc}
0.7 & 0 \\
0 & 0.6
\end{array}\right) \text {, } \\
& \mathscr{B}_{11}=\left(\begin{array}{cc}
-0.03+0.08 i & -0.05+0.02 i \\
-0.03-0.02 i & 0.08+0.02 i
\end{array}\right) \text {, } \\
& \mathscr{B}_{12}=\left(\begin{array}{cc}
-0.04-0.02 i & -0.04+0.02 i \\
-0.04-0.03 i & 0.06+0.04 i
\end{array}\right) \text {, } \\
& \mathscr{B}_{13}=\left(\begin{array}{rr}
-0.05+0.07 i & -0.02+0.05 i \\
-0.05-0.08 i & -0.05+0.02 i
\end{array}\right) \text {, } \\
& \mathscr{C}_{11}=\left(\begin{array}{ll}
0.5-0.4 i-0.3+0.3 i \\
0.4+0.3 i-0.7-0.9 i
\end{array}\right) \text {, } \\
& \mathscr{C}_{12}=\left(\begin{array}{cc}
0.6-0.5 i-0.4+0.4 i \\
0.5+0.4 i-0.8-0.8 i
\end{array}\right) \text {, } \\
& \mathscr{C}_{13}=\left(\begin{array}{ll}
0.7-0.6 i & -0.5+0.5 i \\
0.6+0.5 i & -0.9-0.7 i
\end{array}\right) \text {, } \\
& \mathscr{D}_{11}=\left(\begin{array}{cc}
0.4-0.4 i & 0.2-0.2 i \\
-0.4+0.3 i & 0.3+0.2 i
\end{array}\right) \text {, } \\
& \mathscr{D}_{12}=\left(\begin{array}{cc}
0.5-0.5 i & -0.4-0.5 i \\
-0.5+0.4 i & 0.4+0.3 i
\end{array}\right) \text {, } \\
& \mathscr{D}_{13}=\left(\begin{array}{ll}
-0.5-0.6 i & 0.4-0.4 i \\
-0.6+0.5 i & 0.5+0.4 i
\end{array}\right) \text {, } \\
& \mathscr{E}_{11}=\left(\begin{array}{cc}
-0.4-0.6 i & 0.3-0.5 i \\
-0.6+0.5 i & 0.5-0.6 i
\end{array}\right) \text {, } \\
& \mathscr{E}_{12}=\left(\begin{array}{cc}
-0.3-0.6 i & 0.6-0.3 i \\
-0.7+0.5 i & 0.3-0.5 i
\end{array}\right) \text {, } \\
& \mathscr{E}_{13}=\left(\begin{array}{cc}
-0.5-0.4 i & 0.5-0.5 i \\
-0.4+0.5 i & 0.4-0.5 i
\end{array}\right) \text {, } \\
& \mathscr{E}_{21}=\left(\begin{array}{cc}
-0.5-0.6 i & 0.4-0.2 i \\
-0.6+0.5 i & 0.5-0.7 i
\end{array}\right) \text {, } \\
& \mathscr{E}_{22}=\left(\begin{array}{cc}
0.7-0.8 i & -0.4-0.3 i \\
-0.6+0.5 i & 0.1-0.4 i
\end{array}\right) \text {, }
\end{aligned}
$$

$$
\begin{aligned}
& \mathscr{C}_{23}=\left(\begin{array}{cc}
-0.2-0.5 i & 0.4-0.1 i \\
-0.5+0.5 i & 0.6+0.2 i
\end{array}\right), \\
& \mathscr{C}_{21}=\left(\begin{array}{cc}
1.2-1.5 i & -1.6+0.3 i \\
1.5+0.4 i & 1.6-0.4 i
\end{array}\right), \\
& \mathscr{C}_{22}=\left(\begin{array}{cc}
1.3-0.4 i & -1.4+0.6 i \\
1.8+0.5 i & 1.3-0.7 i
\end{array}\right), \\
& \mathscr{C}_{23}=\left(\begin{array}{cc}
1.4-1.3 i & -1.3+0.2 i \\
1.4+0.6 i & 1.4-0.6 i
\end{array}\right), \\
& \mathscr{D}_{21}=\left(\begin{array}{cc}
0.5-0.4 i & -0.3+0.3 i \\
-0.4-0.3 i & 0.3-0.3 i
\end{array}\right), \\
& \mathscr{D}_{22}=\left(\begin{array}{cc}
0.6-0.5 i & -0.4+0.4 i \\
-0.5-0.4 i & 0.4-0.4 i
\end{array}\right), \\
& \mathscr{D}_{23}=\left(\begin{array}{cc}
0.7-0.6 i & -0.5+0.5 i \\
-0.6-0.5 i & 0.5-0.5 i
\end{array}\right), \\
& \mathscr{H}_{11}=\left(\begin{array}{cc}
-0.4-0.6 i & 0.4-0.4 i \\
-0.6+0.5 i & 0.8-0.9 i
\end{array}\right), \\
& \mathscr{H}_{12}=\left(\begin{array}{cc}
0.5-0.2 i & 0.3+0.4 i \\
-0.4+0.5 i & 0.3-0.4 i
\end{array}\right), \\
& \mathscr{H}_{13}=\left(\begin{array}{cc}
-0.5-0.6 i & 0.4-0.2 i \\
-0.1+0.5 i & 0.3-0.2 i
\end{array}\right),
\end{aligned}
$$

$\Pi$ represents the partly unknown transition rate matrix, which is considered as

$$
\Pi=\left[\begin{array}{ccc}
-6 & ? & ? \\
5 & ? & ? \\
? & ? & -4
\end{array}\right] .
$$

In the following, a $H \infty$ controller will be designed based on Theorem 1, where the activation function is set as $\varphi(\vartheta(t))=\tanh (\vartheta(t))+i \tanh (\vartheta(t)), \quad \sigma=0.4, \sigma_{d}=0.1, \quad L=$ $\left[\begin{array}{llll}1 & 0 ; 0 & 1\end{array}\right]$, and $\sigma(t)=t-(0.3+0.1 * \sin (t))$ expresses the time-varying delay. If the initial value is set as $\psi(t)=[-0.3+0.5 i ; 0.5-0.3 i]$, the solution could be obtained by the LMI toolbox, and $\gamma=7.6623$ is obtained, the corresponding controller gain matrices are computed as

$$
\begin{aligned}
& \mathscr{K}_{1}=\left(\begin{array}{cc}
9.4442-38.5585 i & -10.3759+26.9077 i \\
21.1045-23.0361 i & -31.4861-1.6866 i
\end{array}\right), \\
& \mathscr{K}_{2}=\left(\begin{array}{cc}
-7.6922-13.4025 i & 16.2192+6.3072 i \\
4.0202-11.3110 i & 5.6539-22.0753 i
\end{array}\right), \\
& \mathscr{K}_{3}=\left(\begin{array}{cc}
-4.3350-31.0012 i & 4.9612+25.3198 i \\
-10.1567-23.3295 i & -13.8700+12.1124 i
\end{array}\right),
\end{aligned}
$$

and then system (5) is stochastically stable under the controllers $u(t)=\mathscr{K}_{p} \vartheta(t), p \in\{1,2,3\}$, and the states are shown as in Figures 1 and 2 .

Figures 3 and 4 depict the imaginary part and real part of the states of $u(t)$, respectively, Figures 1 and 2 depict the states of the imaginary and the real part, respectively, via 


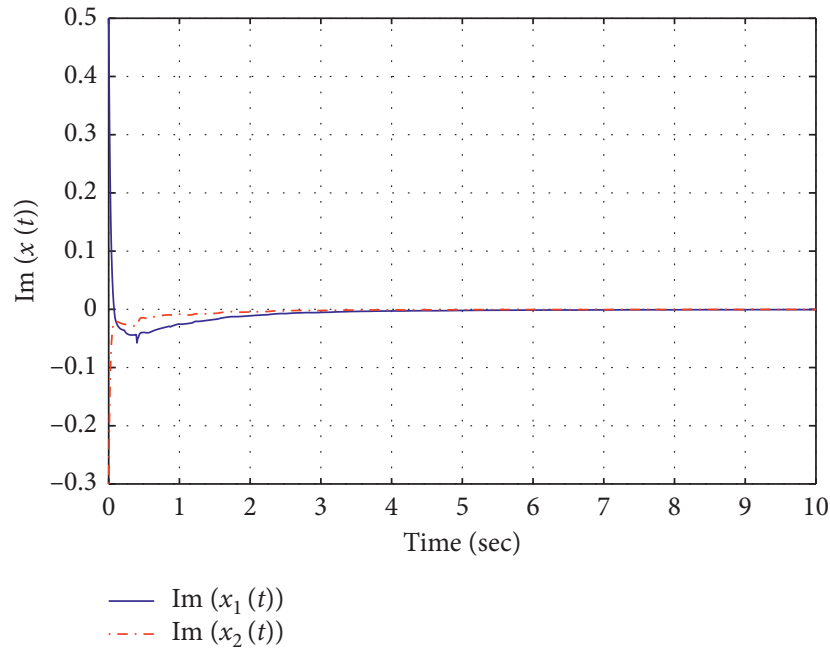

FIgURE 1: Responses the state of the imaginary part.

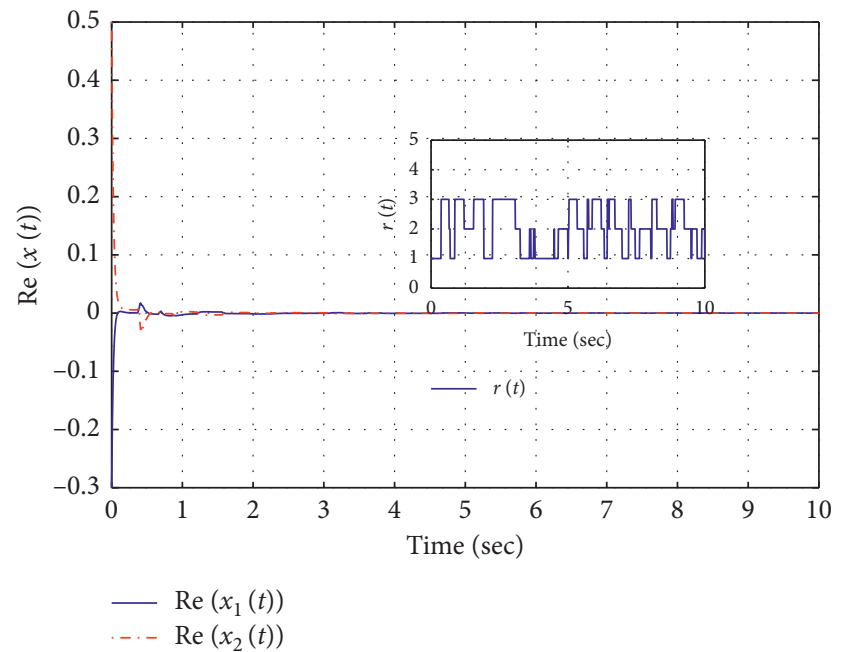

Figure 2: Responses the state of the real part.

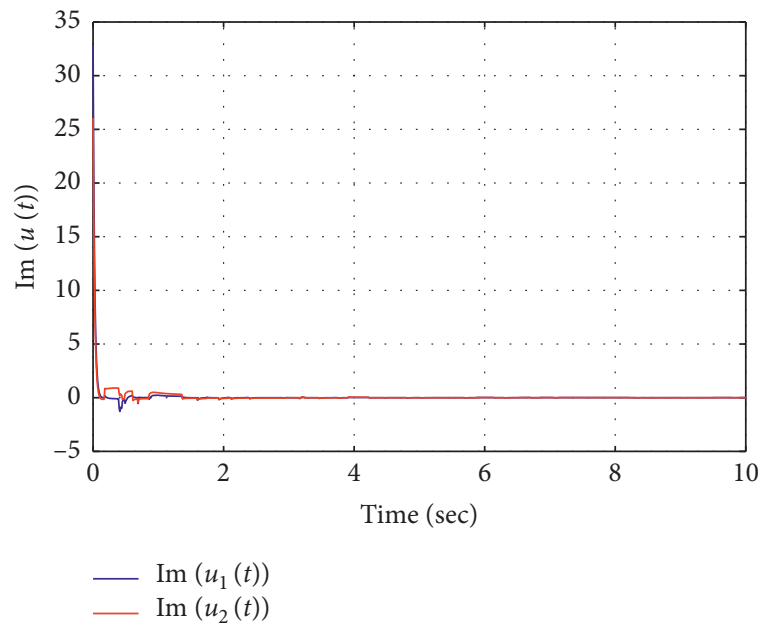

FIgURE 3: Responses input $u(t)$ of the imaginary part.

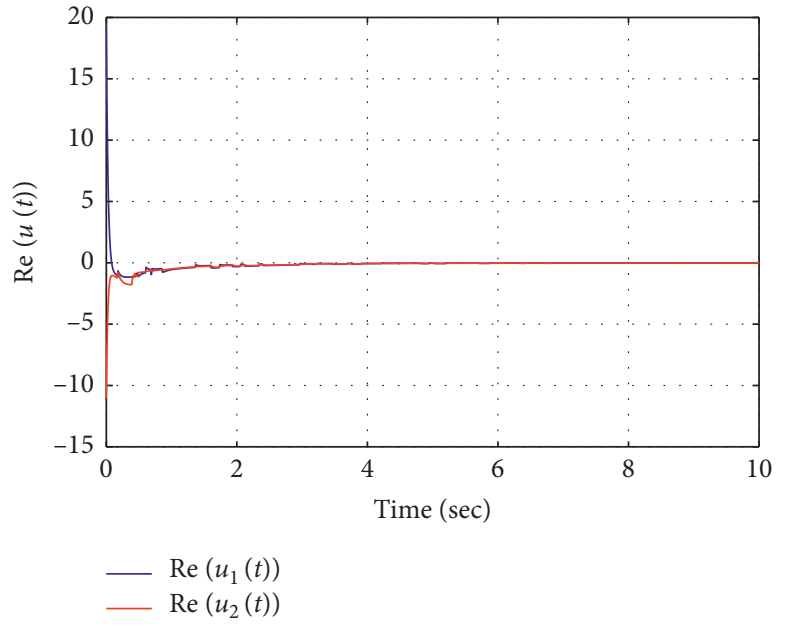

Figure 4: Responses control input $u(t)$ of the real part.

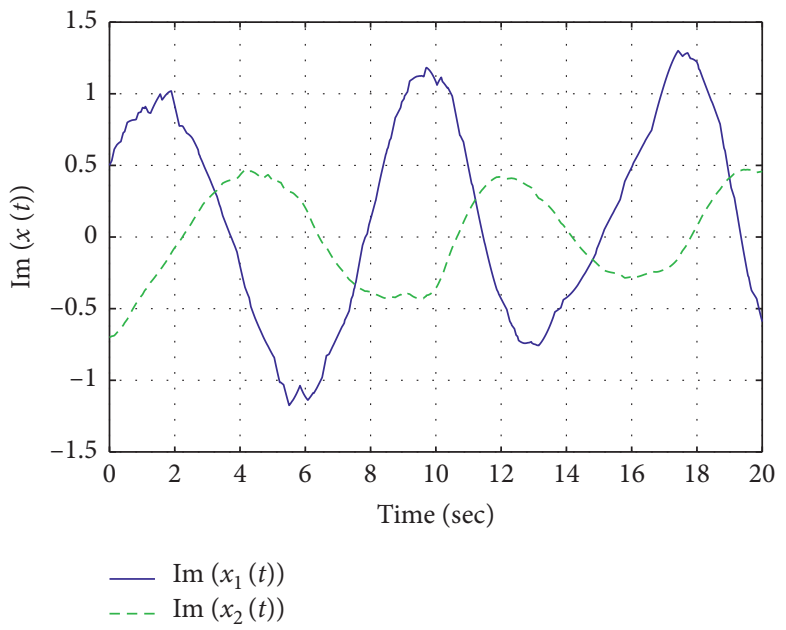

FIgURE 5: The imaginary part of the state without $u(t)$.

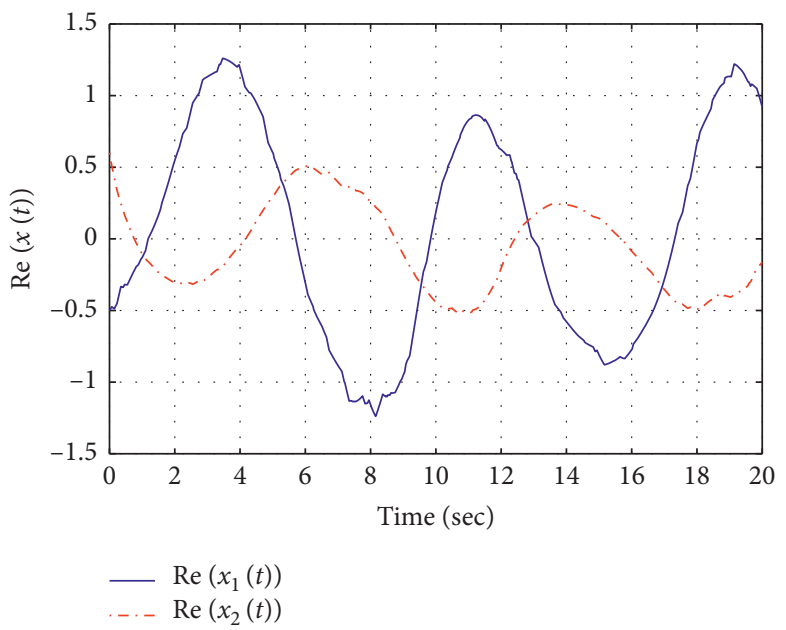

Figure 6: The real part of the state without $u(t)$. 


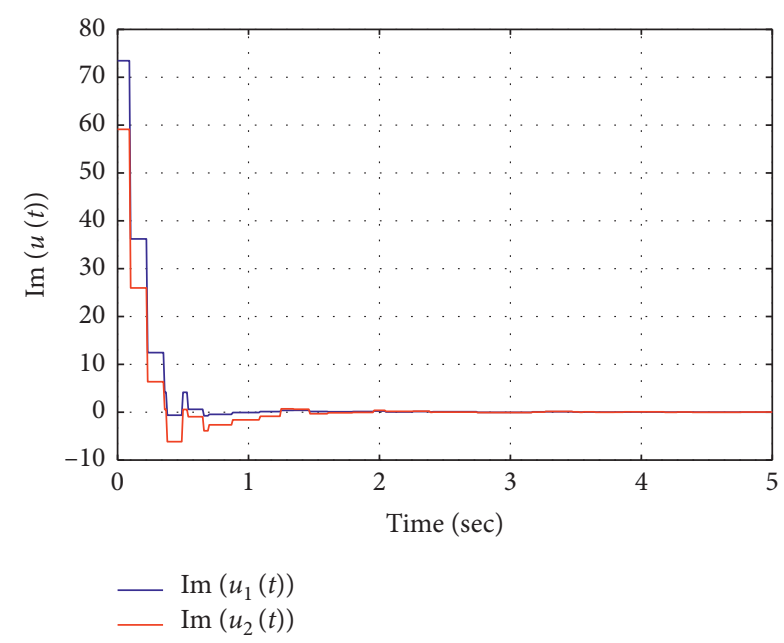

FIgURE 7: Responses input $u(t)$ of the imaginary part.

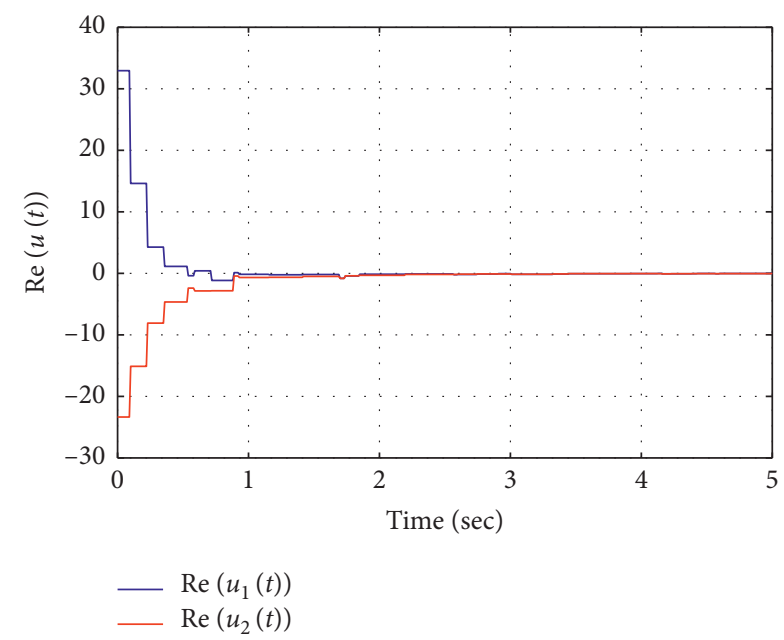

FIGURE 8: Responses control input $u(t)$ of the real part.

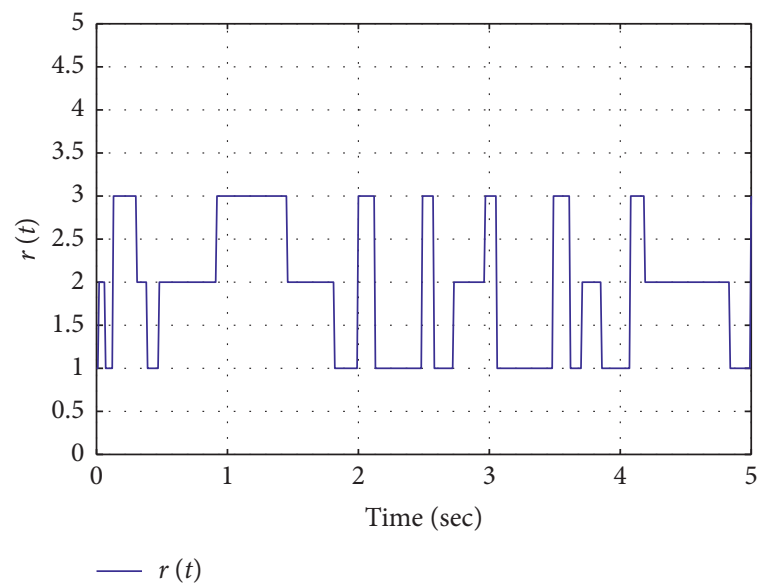

FIGURE 9: Markov signal.

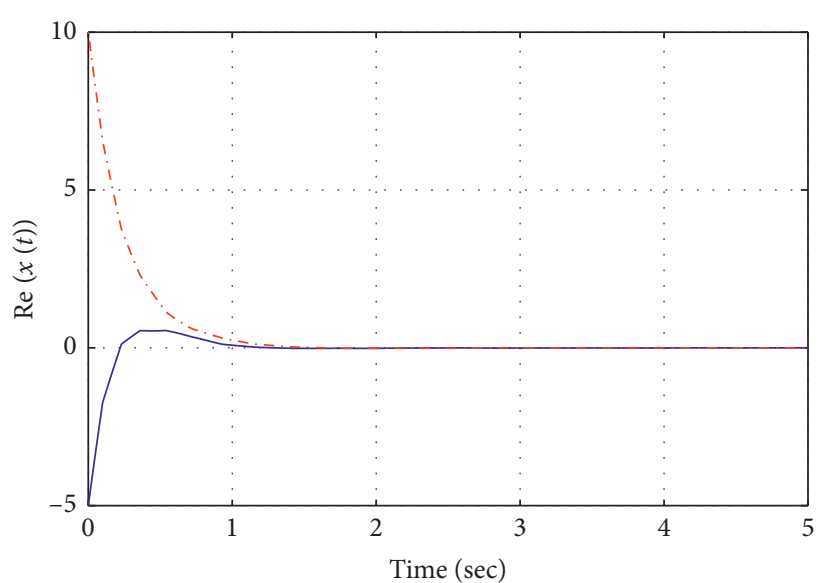

$-\operatorname{Re}\left(x_{1}(t)\right)$

-. $\operatorname{Re}\left(x_{2}(t)\right)$

Figure 10: Responses the state of the real part.

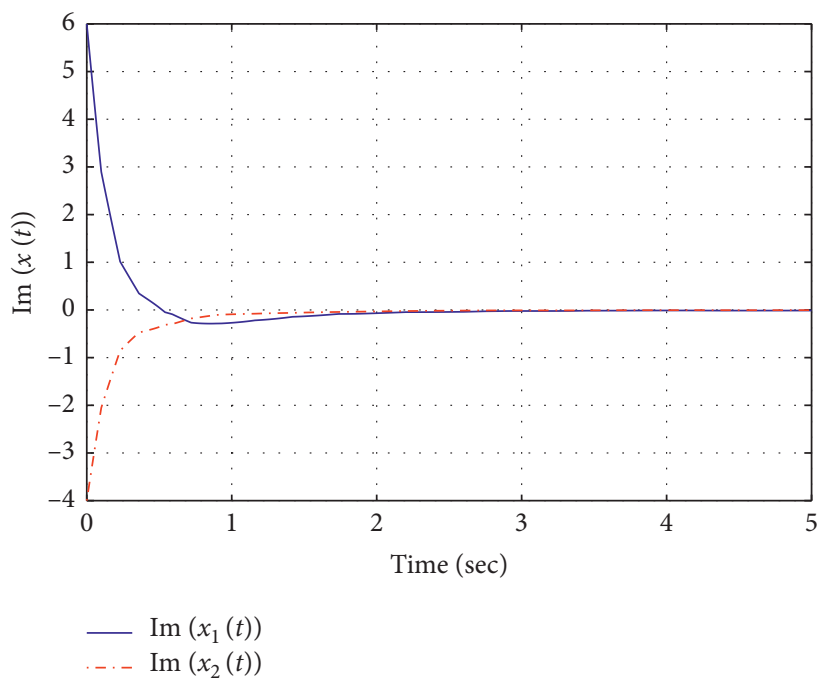

Figure 11: Responses the state of the imaginary part.

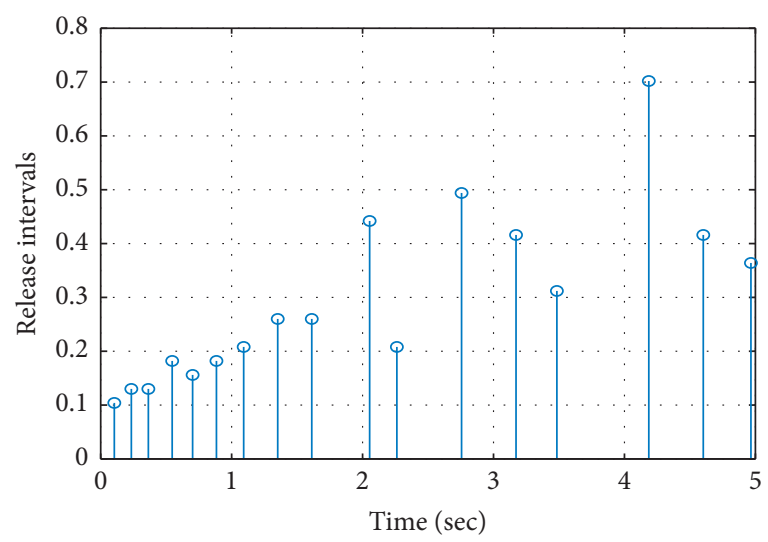

FIgURE 12: The interval of the event-triggered. 
applying the controller, and also, the signal of Markov jump is represented in (7). Compared with Figures 5 and 6 which are the state of Example 1 without controller, it is clear that the controller we designed can stabilize an unstable system effectively.

Example 2. For the same system parameters with Example 1, $\sigma(t)=t-(0.3-0.1 \sin (t))$ expresses the time-varying delay, and $\sigma_{d}=0.1$, set $\Phi=0.2, h=0.036, \varsigma=0.01$, and $\rho=20$, with the external disturbance $\omega=\left(1 / 1-t^{2}\right)$. When the initial value is set as $\psi(t)=[-5+6 i ; 10-4 i]$ that the SBET weighted matrix and control gain matrices are obtained,

$$
\begin{aligned}
\widehat{\mathscr{G}} & =10^{3} \times\left(\begin{array}{cc}
4.1883+0.0000 i & -0.6747-0.2273 i \\
-0.6747+0.2273 i & 3.1574+0.0000 i
\end{array}\right), \\
\widetilde{\mathscr{K}}_{1} & =\left(\begin{array}{cc}
0.9748-6.5659 i & -1.3351+2.9435 i \\
3.3333-4.7188 i & -3.5523+0.1313 i
\end{array}\right), \\
\widetilde{\mathscr{K}}_{2} & =\left(\begin{array}{cc}
-1.2784-2.0467 i & 1.6042+0.7371 i \\
1.5378-2.2989 i & 0.1075-2.3443 i
\end{array}\right), \\
\widetilde{\mathscr{K}}_{3} & =\left(\begin{array}{cc}
-1.2773-5.5737 i & 0.3576+3.1295 i \\
-2.5166-4.9823 i & -1.6298+1.9885 i
\end{array}\right) .
\end{aligned}
$$

Under the conditions which are given in this example, the imaginary part and real part of control inputs $u(t)$ are portrayed in Figures 7 and 8 and Figure 9 is the Markov signals. Figures 10 and 11 are given to show the states of the real part and imaginary part, respectively. It is easy to see system (8) is tending towards stability soon under the controllers which are designed in Theorem 2. Figure 12 shows the release instants of the controller clearly. With the sample interval $h=0.036$, it is not difficult to find that the number of data transfers is reduced dramatically by comparing to the time-driven one.

\section{Conclusions}

In this work, the stability and stabilization of a class of neutral-type CVNNs with time-varying delay and partly unknown Markov jump have been addressed. After designing a $H \infty$ state-feedback control to investigate the stabilization of our system, we have studied the stabilization of CVNNs with time-varying delay and partly unknown Markov jump by a sampling-based event-triggered controller for the first time. Moreover, the feasibility and validity of our results have been proved through two examples. However, there are also some issues that need further consideration, for instance, to design a new eventtriggered to reduce the number of data-sampling transmission and to develop novel LKFs to reduce the conservatism of the stabilization conditions, and the derivation of less conservative integral inequalities can also help us to get better results; they will put into practice in our work in the near future.

\section{Data Availability}

No data were used to support this study.

\section{Conflicts of Interest}

The authors declare that they have no conflicts of interest.

\section{Acknowledgments}

This work was supported by the National Nature Science Foundation of China under grant/award no. 12061088.

\section{References}

[1] Y. Kao, Y. Li, J. H. Park et al., "Mittag-leffler synchronization of delayed fractional memristor neural networks via adaptive control," IEEE Transactions on Neural Networks and Learning Systems, vol. 99, pp. 1-6, 2020.

[2] X. Ji, J. Lu, J. Lou et al., "A unified criterion for global exponential stability of quaternion-valued neural networks with hybrid impulses," International Journal of Robust and Nonlinear Control, vol. 30, pp. 8098-8116, 2020.

[3] L. Hua, S. Zhong, K. Shi, and X. Zhang, "Further results on finite-time synchronization of delayed inertial memristive neural networks via a novel analysis method," Neural Networks, vol. 127, pp. 47-57, 2020.

[4] J. Guo, Z. Meng, and Z. Xiang, "Passivity analysis of stochastic memristor-based complex-valued recurrent neural networks with mixed time-varying delays," Neural Processing Letters, vol. 47, no. 3, pp. 1097-1113, 2018.

[5] R. Samidurai, R. Sriraman, J. Cao, and Z. Tu, "Eects of leakage delay on global asymptotic stability of complex-valued neural networks with interval time-varying delays via new complexvalued Jensens inequality," International Journal of Adaptive Control and Signal Processing, vol. 32, no. 9, pp. 1294-1312, 2018.

[6] L. Li, Z. Wang, Y. Li, H. Shen, and J. Lu, "Hopf bifurcation analysis of a complex-valued neural network model with discrete and distributed delays," Applied Mathematics and Computation, vol. 330, pp. 152-169, 2018.

[7] D. Liu, S. Zhu, and W. Chang, "Input-to-state stability of memristor-based complex-valued neural networks with time delays," Neurocomputing, vol. 221, pp. 159-167, 2017.

[8] J. Hu and J. Wang, "Global stability of complex-valued recurrent neural networks with time-delays," IEEE Transactions on Neural Networks and Learning Systems, vol. 23, no. 6, pp. 853-865, 2012.

[9] Z. Zhang and S. Yu, "Global asymptotic stability for a class of complex-valued Cohen Grossberg neural networks with time delays," Neurocomputing, vol. 171, pp. 1158-1166, 2015.

[10] X. Xu, J. Zhang, and J. Shi, "Exponential stability of complexvalued neural networks with mixed delays," Neurocomputing, vol. 128, pp. 483-490, 2014.

[11] Y. Yuan, Q. Song, Y. Liu, and F. E. Alsaadi, "Synchronization of complex-valued neural networks with mixed two additive timevarying delays," Neurocomputing, vol. 332, pp. 149-158, 2019.

[12] J. Liang, K. Li, Q. Song, Z. Zhao, Y. Liu, and F. E. Alsaadi, "State estimation of complex-valued neural networks with two additive time-varying delays," Neurocomputing, vol. 309, pp. 54-61, 2018.

[13] V. Vembarasan, P. Balasubramaniam, and E. M. Joo, " $H \infty$ state-feedback control of time-delay systems using 
reciprocally convex approach," Journal of Process Control, vol. 24, no. 6, pp. 892-904, 2014.

[14] J. Xiong, J. Lam, H. Gao, and D. W. C. Ho, "On robust stabilization of Markovian jump systems with uncertain switching probabilities," Automatica, vol. 41, no. 5, pp. 897-903, 2005.

[15] K. Shi, J. Wang, S. Zhong, Y. Tang, and J. Cheng, "Non-fragile memory filtering of T-S fuzzy delayed neural networks based on switched fuzzy sampled-data control," Fuzzy Sets and Systems, vol. 394, pp. 40-64, 2020.

[16] X. Cai, S. Zhong, J. Wang, and K. Shi, "Robust Ho control for uncertain delayed T-S fuzzy systems with stochastic packet dropouts," Applied Mathematics and Computation, vol. 385, Article ID 125432, 2020.

[17] H. Shen, F. Li, H. Yan, H. R. Karimi, and H.-K. Lam, "Finitetime event-triggered $\$ \backslash$ mathcal $\{\mathrm{H}\}_{-}\{$\infty $\}$\$ control for T-S fuzzy markov jump systems," IEEE Transactions on Fuzzy Systems, vol. 26, no. 5, pp. 3122-3135, 2018.

[18] X. H. Chang, Robust Output Feedback Ho Control and Filtering for Uncertain Linear Systems, Springer Science Business, Berlin, Germany, 2014.

[19] C. Huang, Y. Bai, and X. Liu, "H1state feedback control for a class of networked cascade control systems with uncertain delay," IEEE Transactions on Industrial Informatics, vol. 6, no. 1, pp. 62-72, 2010.

[20] X. H. Chang, "Ho controller design for linear systems with time-invariant uncertainties," International Journal of Control, Automation and Systems, vol. 9, no. 2, pp. 1-5, 2011.

[21] L. Xiong, H. Zhang, Y. Li et al., "Improved stability and $H_{\infty}$ performance for neutral systems with uncertain markovian jump," Nonlinear Analysis, Hybrid Systems, vol. 19, pp. 13-25, 2016.

[22] Y. Kang, Y. B. Zhao, and P. Zhao, Stability Analysis of Markovian Jump Systems, Science Press, Beijing, Germany, 2018.

[23] X. Liu and H. Xi, "Stability analysis for neutral delay markovian jump systems with nonlinear perturbations and partially unknown transition rates," Advances in Mathematical Physics, vol. 2013, Article ID 592483, 20 pages, 2013.

[24] Y. Zhang, Y. He, M. Wu, and J. Zhang, "Stabilization for Markovian jump systems with partial information on transition probability based on free-connection weighting matrices," Automatica, vol. 47, no. 1, pp. 79-84, 2011.

[25] A. C. Sowmiya, B. R. Raja, C. Q. Zhu et al., "Further meansquare asymptotic stability of impulsive discrete-time stochastic BAM neural networks with markovian jumping and multiple time-varying delays," Journal of the Franklin Institute, vol. 356, no. 1, pp. 561-591, 2019.

[26] X. Mao, "Stability of stochastic differential equations with markovian switching," Stochastic Processes and Their Applications, vol. 79, no. 1, pp. 45-67, 1999.

[27] L. Xiong, J. Tian, and X. Liu, "Stability analysis for neutral markovian jump systems with partially unknown transition probabilities," Journal of the Franklin Institute, vol. 349, no. 6, pp. 2193-2214, 2012.

[28] Z. Wu, P. Shi, H. Su, and J. Chu, "Stochastic synchronization of markovian jump neural networks with time-varying delay using sampled data," IEEE Transactions on Cybernetics, vol. 46, no. 3, pp. 1796-1806, 2013.

[29] H. Zhang, J. Wang, Z. Wang, and H. Liang, "Sampled-data synchronization analysis of markovian neural networks with generally incomplete transition rates," IEEE Transactions on Neural Networks and Learning Systems, vol. 28, no. 3, pp. 740-752, 2017.
[30] C. Zhao, S. Zhong, X. Zhang et al., "Novel results on nonfragile sampled-data exponential synchronization for delayed complex dynamical networks," International Journal of Robust and Nonlinear Control, vol. 30, no. 10, pp. 4022-4042, 2020.

[31] P. Tabuada, "Event-triggered real-time scheduling of stabilizing control tasks," IEEE Transactions on Automatic Control, vol. 52, no. 9, pp. 1680-1685, 2007.

[32] W. P. M. H. Heemels, J. H. Sandee, and P. P. J. Van Den Bosch, "Analysis of event-driven controllers for linear systems," International Journal of Control, vol. 81, no. 4, pp. 571-590, 2008.

[33] C. Peng and J. Zhang, "Event-triggered output-feedback $H \infty$ control for networked control systems with time-varying sampling," IET Control Theory \& Applications, vol. 9, no. 9, pp. 1384-1391, 2015.

[34] D. Yue, E. Tian, and Q.-L. Han, "A delay system method for designing event-triggered controllers of networked control systems," IEEE Transactions on Automatic Control, vol. 58, no. 2, pp. 475-481, 2013.

[35] R. Zhang, D. Zeng, S. Zhong, and Y. Yu, "Event-triggered sampling control for stability and stabilization of memristive neural networks with communication delays," Applied Mathematics and Computation, vol. 310, pp. 57-74, 2017.

[36] K. J. Astrom and B. Bo, "Comparison of periodic and event based sampling for first order stochastic systems," in Proceedings of IFAC World Congress, pp. 301-306, Elsevier, Beijing, China, September 1999.

[37] X. Wang, Z. Wang, Q. Song, H. Shen, and X. Huang, "A waiting-time-based event-triggered scheme for stabilization of complex-valued neural networks," Neural Networks, vol. 121, pp. 329-338, 2020.

[38] Z. Wang, L. Shen, J. Xia, H. Shen, and J. Wang, "Finite-time non-fragilel2-locontrol for jumping stochastic systems subject to input constraints via an event-triggered mechanism," Journal of the Franklin Institute, vol. 355, no. 14, pp. 6371-6389, 2018.

[39] C. Peng and T. C. Yang, "Event-triggered communication and Ho control co-design for networked control systems," Automatica, vol. 49, no. 5, pp. 1326-1332, 2013.

[40] C. Hu, H. Yan, H. Zhang, and S. Chen, "Event-triggered output feedback $H \infty$ control for networked control systems with time-varying sampling and packet losses," in Proceedings of the Chinese Control Conference, pp. 8002-8007, Dalian, China, July 2017.

[41] A. Seuret and F. Gouaisbaut, "Wirtinger-based integral inequality: application to time-delay systems," Automatica, vol. 49, no. 9, pp. 2860-2866, 2013.

[42] C.-A. Popa, "Global $\mu$-stability of neutral-type impulsive complex-valued BAM neural networks with leakage delay and unbounded time-varying delays," Neurocomputing, vol. 376, no. 1, pp. 73-94, 2020. 